Philippe Lacour, Frankfurt/Main

\title{
Diskursivität. Zur logischen Erklärung der Hermeneutik Ricoeurs
}

\begin{abstract}
This article explores the logical dimensions of Ricoeur's definition of Discursivity. This is a key aspect for understanding not only the general meaning of Hermeneutics, but also the particular relevance of the concept in the author's practical philosophy. Ricoeur developed his theory of Discourse after introducing a semiologic turn to his Phenomenological work. Discourse is not reduced to immediate (dialogical) communication, even though it begins with it. Taken as a principle of discursive historicity, text plays a major role in the constitution of this theory - it is considered as a model for detachment in communication. Ricoeur distinguishes different levels of meaning (word, sentence, text, culture) and separates three discursive dimensions, irreducible to one another: Rhetorics, Poetics and Hermeneutics. At this point, the article should explain the role of Hermeneutics, particularly when compared to the other two disciplines. The article will conclude by underlining the role of Reflexivity and the analogical unity of Discursivity. ${ }^{1}$
\end{abstract}

\section{Key words}

Discourse, Communication, Distanciation, Text, Rhetorics, Poetics, Hermeneutics, Reflexivity, Dialogue, Work.

\section{Einleitung}

In diesem Artikel versuche ich zu erklären, auf welchen logischen Gründen die Definition der Diskursivität bei Ricoeur basiert. Nur so können sowohl die Bedeutung der Hermeneutik im allgemeinen als auch ihr Stellenwert für die ganze praktische Philosophie Ricoeurs nachvollzogen werden ${ }^{2}$. Unter dem Begriff „Logik“ versteht Ricoeur nicht die formale Logik sondern in eher traditioneller Art und Weise die allgemeinen Eigenschaften des Logos, des Diskurses.

Den Diskurs definiert Ricoeur auf folgende Weise: „Sprechen“ bedeutet für ihn, dass jemand etwas über etwas - phonetischen, lexikalischen, syntaktischen und stilistischen Regeln gemäß - einem anderen sagt ${ }^{\star 3}$, wobei für ihn allerdings die aktuelle gegenwärtige Diskussion nur ein Teil des Diskurses ist. Eigentlich sollte eine ernst zu nehmende und zusammenhängende Theorie des Diskurses in der Lage sein, diese erste Dimension mit den anderen (z.B. den poetischen) Aspekten der Sprache in Verbindung zu bringen, und so scheint Ricoeur tatsächlich von Anfang an verstanden zu haben, dass der Diskurs, auch wenn er mit der unmittelbaren Kommunikation ${ }^{4}$ beginnt, nicht auf sie reduziert werden kann ${ }^{5}$.

1 Ich möchte Andris Breitling, Emmanuel Prokob und Marie Reetz für Ihre Hilfe danken.

2 Vier unübersetzte Texte von Ricoeur können dabei helfen, diese logische Untersuchung zu verstehen: 1) „Discours et communication“, in La Communication. Actes du XV $V^{e}$ Congrès de l'association des sociétés de philosophie de langue française. Université de Montréal, 1971, Montréal 1973 (auch in: Paul Ricoeur, L'Herne, 2004, S. 51-67); 2) „La fonction herméneutique de la distanciation“, in Du texte à l'action (Paris, Seuil, 1986); 3) „Rhétorique, poétique, herméneutique“, in Lectures 2 (Paris, Seuil, 1999); 4) Sur la traduction (Paris, Bayard, 2004) - und vielleicht auch das Vorwort von Réflexion faite, Paris, Esprit, 1995.

3 Siehe „Eine intellektuelle Autobiographie“ in Vom Text zur Person. Hermeneutische Aufsätze (1970-1999) (übers. und hrsg. Von Peter Welsen, Felix Verlag 2005), S. 32.

4 Im Gegensatz zur unmittelbaren, synchronen und direkten Kommunikation des Dialogs, die in der gegenwärtigen Zeit stattfindet, kann die mittelbare Kommunikation als Kommunikation mit zeitlicher Distanz bezeichnet werden.

5 Vielleicht liegt der größte Unterschied zwischen Ricoeur und Habermas darin, dass sie eine unterschiedliche 
Um diese Theorie des Diskurses zu verstehen, muss man Ricoeurs Gedankengang durch verschiedene Etappen hindurch folgen, angefangen bei der semiotischen Wende seiner Phänomenologie, die die Hermeneutik als sinnbildende Reflexion einstuft (im Gegensatz zu der Hermeneutik des Symbols/Geheimnisses/Doppelsinns) (1). Im Anschluss lässt sich die allgemeine Definition des Diskurses erklären (2), bei deren Bildung dann der Text als Geschichtlichkeitsprinzip des Diskurses eine große Rolle spielt - der Text wird hier als Modell der Distanzierung in der Kommunikation verstanden (3). Außerdem müssen die verschiedenen Sinnesebenen (Wort, Satz, Text, Kultur) unterschieden werden (4). Es muss erwähnt werden, dass Ricoeur innerhalb der Sprache drei verschiedene, miteinander unvereinbare Dimensionen des Diskurses unterscheidet; woraus sich dann die Stellung der Hermeneutik neben der Rhetorik und der Poetik (5) ergibt. Zuletzt werden die Rolle der Reflexivität und die analogische Einheit der Diskursivität besprochen (6).

\section{Die semiotische Wende der Phänomenologie Ricoeurs}

Die Interpretationstheorie von Ricoeur hat zu einem neuartigen Hermeneutikbegriff geführt (der sogenannten „Hermeneutik der Distanzierung“). Um sie zu verstehen, muss man zunächst ihre Entstehung im Lichte der Beziehung Ricoeurs zu Husserls Phänomenologie betrachten, wobei Ricoeur sich dieser mal annähert, und dann wieder von ihr abweicht ${ }^{6}$. Erstens wurzelt die Hermeneutik in der Phänomenologie, insofern als es eine allgemeine Verwandtschaft zwischen dem Textverständnis und der intentionalen Beziehung eines Bewusstseins zu einem ihm gegenüberstehenden Sinn gibt. Zweitens wird das Thema der Lebenswelt, das für die post-heideggerianische Hermeneutik zentral ist, von der Phänomenologie Husserls zwar entdeckt, aber doch vernachlässigt und teilweise negiert ${ }^{7}$.

In der dritten der genannten Beziehungen weicht die Hermeneutik am meisten von der Phänomenologie ab. Für Husserl wird das Selbstverständnis des Bewusstseins durch eine unmittelbare und einfache, in Wahrheit aber nur scheinbare Reduktion erreicht. Für Ricoeur dagegen wird das Selbstverständnis des Bewusstseins durch die mühsame, aber produktive Vermittlung von sprachlichen Zeichen, Symbolen (als doppelsinnige Ausdrücke in traditionellen Kulturen $)^{8}$ und Texten ${ }^{9}$ ermöglicht.

Die Ursache für diesen Unterschied liegt in der Bedeutungsänderung der Reduktion. Dabei kann man von einer semiotischen Wende in der Phänomenologie Ricoeurs sprechen.

Definition des Diskurses benutzen, um den erneuerten Begriff der praktischen Vernunft zu definieren. Um diesen Unterschied zusammenzufassen, könnte man sagen, dass für Habermas der Diskurs mit der unmittelbaren Kommunikation zusammen gelegt wird, aber nicht für Ricoeur, der die unmittelbare Kommunikation nur als fundamentales Niveau des Diskurses nennt.

6 Siehe Du texte à l'action, Paris, Seuil, 1986, S.7: „Die Hermeneutik - oder allgemeine Theorie der Interpretation- hat mit der Auseinandersetzung mit der husserlschen Phänomenologie nie aufgehört. Sie entwickelt sich daraus in einem doppelten Sinn: die Phänomenologie ist der Ort, von dem sie herkommt, und auch der Ort, den sie verlassen hat".

7 Siehe "De l'interprétation", in Du texte à l'action, ebd., S. 32: "Le thème de la Lebenswelt, rencontré en quelque sorte contre son gré par la phénoménologie, est assumé par l'herméneutique post-heideggérienne non plus comme un résidu mais comme un préalable""'.

8 Du texte à l'action, ebd., S. 34. Man kann aber die Hermeneutik nicht allein durch die symbolische Auslegung bestimmen, wie Ricoeur es einmal betrachtete: erstens weil der traditionelle Symbolismus seine Sinnvervielfältigung nur in angepassten Kontexten entfaltet, d. h. im Maßstab des ganzen Textes; zweitens weil aus dem gleichen Symbolismus konkurrierende Auslegungen herauskommen, und weil dieser Kampf der Interpretationen sich auch im Maßstab der Texte entfaltet.

9 Du texte à l'action, ebd., S. 35. Die Vermittlung durch die Texte reduziert den Bereich der Interpretation ausschließlich auf den Bereich der Schrift, aber öffnet auch neuartige Perspektiven für den Diskurs, der eine dreimalige semantische Unabhängigkeit bekommt: in Bezug auf die Sprechermeinung, mit der Rezeption durch die primitive Hörerschaft, und mit den wirtschaftlichen, sozialen und kulturellen Bedingungen seiner Produktion. 
So schrieb Ricoeur im Jahr 1969: „Die Symbolfunktion (...) ist keineswegs eine Klasse, eine Gattung, sondern eine Bedingung der Möglichkeit (...) In diesen Begriffen formuliert, scheint mir die Frage nach dem Ursprung der Symbolfunktion eine ganz neue Interpretation der phänomenologischen Reduktion auf den Plan zu rufen : Die Reduktion, würde ich sagen, ist

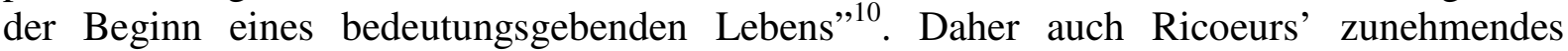
Interesse am Symbolismus, der für ihn in der Interpretation der Symbolik des Bösen und den Produktionen des Unbewussten wurzelt. Hier wäre allerdings anzumerken, dass Ricoeur die verschiedenen Symbolsysteme manchmal auf die Sprache eingeschränkt ${ }^{11}$ hat, ohne dabei zu hinterfragen, welcher Unterschied zwischen formalen Systemen (Mathematik, Logik in ihren verschiedenen Arten), natürlichen (d. h. sprachlichen) Systemen (Deutsch, Französisch) und anderen (Musik, Bilder, usw.) besteht ${ }^{12}$. So bezeichnet er zum Beispiel die Psychoanalyse als Therapie, die sich nur in dem „geschlossenen Feld der Sprache“ entwickelt ${ }^{13}$, und übersieht dabei, dass für die Interpretation manchmal auch verschiedene Zeichenarten benutzt werden können (für Kinder z.B. Bilder, wie die französische Therapeutin Françoise Dolto bemerkte). Wenn er jedoch zu zeigen versucht, wie Zeit u. a. durch Erzählen konstruiert wird, vergisst er meiner Meinung nach zu erwähnen, dass auch anderen Ausdrucksmöglichkeiten diese Fähigkeit zugesprochen werden könnte, so z. B. dem musikalischen Symbolsystem, welches zudem über die besondere Möglichkeit verfügt, Zeit zu artikulieren ${ }^{14}$. Dies ist umso erstaunlicher, wenn man bedenkt, dass Ricoeur selbst betont hatte, dass der lyrische Diskurs die Zeit in einer anderen Art und Weise ausdrückt ${ }^{15}$.

Ricoeurs Interesse galt stets eher der deskriptiven (Scheler, Ingarden) als der idealistischen (Fink) Interpretation der Phänomenologie Husserls ${ }^{16}$ und so verwirft er in seinem ersten Buch (die Philosophie de la volonté) die transzendentale Bedeutung der Reduktion zugunsten der eidetischen Reduktion ${ }^{17}$. Der linguistic turn dieser deskriptiven Phänomenologie zieht die methodologische Veränderung von Ricoeurs Philosophie nach sich, die dieser selbst hervorhebt, wenn er von Phänomeno-logie bzw. linguistischer Phänomenologie spricht ${ }^{18}$. Im Zusammenhang mit dieser Entwicklung innerhalb seines Denkens lässt sich dann auch Ricoeurs Vorstellung von der indirekten Natur der Reflexion (im Gegensatz zur direkten und transparenten wie bei Fichte, Husserl oder Kant und Jean Nabert) verstehen ${ }^{19}$. Ricoeur zufolge vermittelt sich das Selbstverständnis immer durch

10 Siehe „Die Frage nach dem Subjekt angesichts der Herausforderung der Semiologie“, in Hermeneutik und Strukturalismus. Der Konflikt der Interpretationen I, Kösel-Verlag, München, 1973, S. 163.

11 Dabei muss man aber vorsichtig sein, weil Ricoeur 1965 in die „Sprache“ alle Typen des „menschlichen Bedeutens" von der formalen Logik bis zur Mythenanalyse einbezieht (vgl. Die Interpretation. Ein Versuch über Freud, Frankfurt am Main, Suhrkamp, 1974). Daher vielleicht die Zweideutigkeit seiner symbolischen Philosophie, die heutzutage wahrscheinlich erneuert werden sollte.

12 Dieser Reihe von Problemen widmete sich der französische Epistemologe G.-G. Granger in seinem ganzen Werk. Siehe meinen Artikel „Gilles-Gaston. Granger et la Critique de la Raison Symbolique“ (http://www.revue-texto.net/Inedits/Lacour_Granger.html).

13 Siehe Die Interpretation, ebd.

14 Siehe das Buch von Bernard Sève, L'altération musicale, Paris, Seuil, 2004.

15 Vgl. Zeit und Erzählung, Band 3, München, Fink, 1991, Abschluss.

16 Siehe „Eine intellektuelle Autobiographie“, in Vom Text zur Person, S. 14; und seine Einleitung und Kommentar (in den Fußnoten) von Ideen I.

17 Vgl. Philosophie de la volonté I. Le volontaire et l'involontaire, Paris, Aubier, 1950. In „Eine intellektuelle Autobiographie“ (S. 16) präzisiert Ricoeur, dass er die Methode der eidetischen Analyse von Husserl übernahm, aber dass er die Problematik eines aktiven und (zur gleichen Zeit) passiven Subjektes von dem französischen existenzialistischen Philosophen Gabriel Marcel bekam.

18 Siehe „Discours et communication“, a. a. O., S. 66: „,e que nous avons pratiqué, c’est une phénoménologie linguistique“.

19 Nach Ricoeur zeigt sich diese indirekte Weise auch in der Intentionalität der Phänomenologie Husserls. Siehe „De l'interprétation“, in Du texte à l'action, ebd., S. 30: „L'acte de viser quelque chose ne s'atteint lui-même qu'à-travers l'unité identifiable et réidentifiable du sens visé“. 
Zeichen, Symbole oder Texte (dies gilt auch für das Projekt einer Auslegung des Dasein $^{20}$ bei Heidegger).

An dieser Stelle soll erwähnt werden, dass Ricoeur seine Konzeption (Bestimmung) der Hermeneutik allmählich änderte. Anfangs hatte er diese Konzeption (Bestimmung) eingeschränkt und als rein doppelsinnige Auslegung angesehen, wie z.B. in Die Symbolik des Bösen (1960) oder in Die Interpretation. Ein Versuch über Freud (1965) ${ }^{21}$, änderte seine Auffassung dann aber aus zwei Gründen, wodurch er 1986 eine umfassendere Definition der Hermeneutik entwickelte: zum einen stellte er fest, dass ein Symbolismus seine Mehrdeutigkeit nur auf der Ebene des Textes ausdrückt; und zum anderen, dass ebendieser Symbolismus in konkurrierenden Interpretationen betrachtet wird, deren Konflikt einzig auf der Textebene (geschieht) wahrnehmbar ist ${ }^{22}$. Daher geht es bei der Hermeneutik nicht (mehr) um den versteckten (oder auch doppelten) Sinn, wie man es Ricoeur damals jedoch gelegentlich nachsagte, als sein Buch über Freud (1965) erschien.

\section{Eine Definition des Diskurses}

Ich zähle die grundlegenden Polaritäten des Diskurses noch einmal kurz auf: Ereignis und Bedeutung, singuläre Identifikation und allgemeine Prädikation, propositionaler und illokutionärer Akt, Sinn und Referenz, Bezug auf die Wirklichkeit und Bezug auf die Gesprächsteilnehmer. ${ }^{23}$

Nach seinem Buch über Freud versucht Ricoeur seine hermeneutischen Intuitionen weiter zu entwickeln ${ }^{24}$, und zwar vor allem im Bereich der Religion und der Psychoanalyse. Zur gleichen Zeit (um 1970) bemüht er sich aber auch, auf der Basis seines früheren Entwurfes von einer Poetik des Willens ${ }^{25}$ eine handfeste Handlungstheorie auszuarbeiten, die die Einbildungskraft als eine sprachliche Dimension einordnet ${ }^{26}$. Hierfür setzt er bei einer besonderen Handlung an, die er dann als allgemeines Handlungsmodell nutzt, und zwar das Sprechen - wobei Ricoeur wie auch Habermas ${ }^{27}$ eigentlich von einem Akt sprechen.

20 Deswegen soll die Hermeneutik nach Ricoeur den langen Weg durch die Geisteswissenschaften nehmen, ohne behaupten zu können, eine direkte hermeneutische Ontologie zu schaffen. Die Ontologie darf nicht ohne Epistemologie funktionieren (siehe „La tâche de l'herméneutique, en venant de Schleiermacher et de Dilthey“, in Du texte à l'action, a. a. O., S. 105.

21 Im Gegensatz zu Cassirer, der in seiner Philosophie der Symbolischen Formen 'Symbol' sehr weit definiert (als universelle Beziehung zwischen dem Menschen und der Welt), bezeichnet Ricoeur das Symbol in einer eingeschränkten Art und Weise als doppelsinnigen Ausdruck. Siehe Die Interpretation, ebd., S. 25: „Ich beschränke also den Begriff des Symbols bewusst auf die doppel- oder mehrdeutigen Ausdrücke, deren semantische Textur in Wechselbeziehung steht mit der Interpretationsarbeit, die ihren zweiten oder vielfachen Sinn expliziert“.

22 Vgl. „De l'interprétation“, in Du texte à l'action, ebd., S. 34.

23 Siehe „Die Metapher und das Hauptproblem der Hermeneutik“, in: Vom Text zur Person, a. a. O., S. 114.

24 In Hermeneutik und Strukturalismus versucht Ricoeur noch, die Hermeneutik zu verteidigen. Danach, in Du texte à l'action, beschäftigt er sich mehr mit der effektiven Praxis der Hermeneutik.

25 Dieses Projekt war der dritte Band der Philosophie de la volonté, a. a. O.

26 Die Imagination wird nicht als verschwindende Vorstellung (reproduktive Imagination), sondern als entstehende Bedeutung betrachtet. Siehe „L'imagination dans le discours et dans l'action“ die mit einem besonderen Projekt anfängt: „Zu einer allgemeinen Theorie der Imagination“ (vgl. Du Texte à l'action., ebd., S. 237). In diesem Text (1976), wie auch in L'ideologie et l'utopie (Paris, Seuil, 1997; Lectures on Ideology and Utopia (Unterrichten von 1975) Columbia University Press, New York, 1986), verbindet Ricoeur sein Interesse an der praktischen Philosophie mit seine Reflexion über das „logische“ Problem der semantischen Innovation (das in Die Lebendige Metapher und Zeit und Erzählung untersucht wurde) (er spricht von einem Schritt von der Theorie in die Praxis). Daher könnte man bei Ricoeur von einer „Logik der praktischen Vernunft“" (oder Logik des Praktischen) sprechen.

27 Siehe z. B. „Bedeutung der Universalpragmatik“, in K.O. Apel, Sprachpragmatik und Philosophie (Frankfurt, Suhrkamp, 1976), wo Habermas lieber von einem „kommunikativen Handeln“ als vom 
Deswegen muss die praktische Philosophie von Ricoeur mit seiner Theorie des Diskurses zusammengebracht werden. Diese Theorie wurde in einem zentralen Artikel mit dem Titel „Discours et communication“ aufgestellt ${ }^{28}$.

Damals versucht Ricoeur, eine Sprachwissenschaft des Diskurses zu entwickeln; und liest zu diesem Zwecke nach eigenem Bekunden auf Linguistik spezialisierte Autoren: Saussure, den zur Zeit des Strukturalismus für alle geradezu verbindlichen Autoren, aber auch Noam Chomsky, Emile Benveniste, Gustave Guillaume und Roman Jakobson ${ }^{29}$. All diese Sprachwissenschaftler helfen ihm den Begriff der Sprache als strukturiertes Inventar durch einen Begriff der Sprache als strukturierende Operation $\mathrm{zu}$ ersetzen ${ }^{30}$. Die Idee einer Sprachwissenschaft des Diskurses übernimmt Ricoeur von Benveniste ${ }^{31}$. Dass diese Art von Linguistik 1971 noch in ihren Kinderschuhen steckt, ist auf den immensen Einfluss von Saussure zurückzuführen, der zwar die Wichtigkeit der Sprache unterstrich, aber die Rolle der „,parole“ nicht anerkannte ${ }^{32}$. Eigentlich ist bei ihm die ,parole“ ein residueller Begriff, da er bezeichnet, was übrig bleibt, nachdem die Sprache thematisiert worden ist: physischer Ausdruck, individuelle Ausführung, freie Kombination, usw. “33. Ricoeur ist einverstanden mit Benveniste und dessen Versuch, sich ,eine Sprachwissenschaft des Diskurses, die etwas Anderes als eine Pragmatik der Sprache wäre“, vorzustellen. Leider wird dieser Versuch durch die Trennung des Semiologischen von dem Semantischen ${ }^{34}$ geschwächt. Dies wiederum zwingt die Linguistik des Diskurses, sich einzig im Gegensatz zur virtuellen, nicht existenten „Sprache“ zu spezifizieren. Ricoeur aber entscheidet sich (zusammen mit Hagège), den Ansatz von Benveniste umzukehren und den Diskurs nicht als ein zu einem virtuellen System hinzukommendes Ereignis zu betrachten, sondern als echte Effektivität, von der die Sprache abstrahiert wird ${ }^{35}$. Diese neue, in eine neue Richtung weisende Perspektive ist sehr wichtig: sie erlaubt es, eine positive und direkte Reflexion über den Diskurs zu entwickeln.

Das Problem besteht darin, dass der Diskurs von widersprüchlicher Natur ist. Obwohl er einen Sinn und eine Referenz ${ }^{36}$ hat, konstituiert er ein Ereignis, das sich einstellt und wieder verschwindet: „Jeder Diskurs wird als Ereignis ausgeführt, aber als Bedeutung verstanden“.

Zwischenverständnis spricht, weil es dabei um eine auf die Bedeutung zielende Handlung geht, die als Norm der anderen Sozialhandlungen betrachtet wird. Siehe „Was heißt Universalpragmatik?“ in Vorstudien und Ergänzungen zur Theorie des kommunikativen Handelns, Frankfurt, Suhrkamp, S. 353.

28 Ricoeur hat diese Diskurstheorie in der Folge manchmal eingeschränkt, wie z. B. in „Die Metapher und das Hauptproblem der Hermeneutik“, in Vom Text zur Person, ebd., S. 112-114.

29 Siehe „Eine intellektuelle Autobiographie“ in Vom Text zur Person, a. a. O., S. 32.

30 Siehe „Die Struktur, das Wort, das Ereignis“, in Hermeneutik und Strukturalismus, S.116.

31 Siehe E. Benveniste, Probleme der allgemeinen Sprachwissenschaft, Frankfurt am Main, 1977.

32 Ricoeur wusste nicht, dass diese starke Trennung auf Baillys Edition von Saussure zurückzuführen ist, und dass Saussure selbst auch eine Sprachwissenschaft der ,parole“ suchte. Vor kurzem wurde dies von Simon Bouquet durch neue Textanalysen gezeigt (siehe: Introduction à la lecture de Saussure, PUF, 1997; und auch http://www.revue-texto.net/Saussure/Sur_Saussure/Bouquet_Linguist-gen.html).

33 Siehe „Discours et communication“, ebd., S. 54.

34 Siehe E. Benveniste, „La forme et le sens dans le langage“, in Probleme der allgemeinen Sprachwissenschaft. Eine derartige doppelte Sprachwissenschaft wurde von Claude Hagège kritisiert. Siehe Der dialogische Mensch: Sprache - Weltbild - Gesellschaft / aus d. Franz. von Monika Hübner, Reinbek b. Hamburg, Rowohlt, 1987, S. 235.

35 Siehe Ricoeur, „Discours et communication“, a. a. O., S. 55.

36 Während bei Frege der Unterschied zwischen „Sinn“ und „Bedeutung“ besteht, gibt Ricoeur der Übersetzung von P. Geach („Bedeutung“ als „Referenz“) den Vorzug (siehe z. B. „Discours et communication“, ebd., S. 55), im Gegensatz zu der französischen Übersetzung von Claude Imbert (,Sens et dénotation“, Ecrits logiques et philosophiques, Verlag Seuil, 1971). Deswegen hat Peter Welsen recht, wenn er „,signification“ als „Bedeutung“ übersetzt (,Die Metapher und das Hauptproblem der Hermeneutik“, in Vom Text zur Person, ebd., S. 112), obwohl Ricoeur manchmal „sens“ als Synonym für „signification“ benutzt (daher stößt man bei ihm auf eine Mehrdeutigkeit des Wortes „sens“, und zwar als Gegenteil entweder von Ereignis oder von Referenz). Dies ermöglicht Peter Welsen eine eindeutige und strenge semantische Trennung zwischen Ereignis/Bedeutung einerseits, und Sinn/Referenz andererseits. 
Ricoeur spricht daher von der „Aufhebung des Ereignisses innerhalb des Sinns“, durch die das Ereignis vernichtet und gleichzeitig in Absicht eines Sinnes zurückgehalten wird, und betrachtet sie, d.h. die Aufhebung, als Grundlage der Kommunikation ${ }^{37}$. Um dieses Paradox zu erklären, entwickelt Ricoeur eine Theorie des Diskurses, die drei Ebenen der Verarbeitung des Sinnes koordiniert und hierarchisch organisiert ${ }^{38}$. Die erste Ebene, die in Aussagen und einer apophantischen Logik besteht (Ricoeur lässt sich bei seiner Unterscheidung von dem Logischen und dem Psychologischen, sowie dem Logischen und dem Ontologischen von Frege, Husserl und Russell inspirieren), übermittelt den auf seinen Inhalt im Satz beschränkten Sinn. Die zweite Ebene ist die des Ausdrucks und der Logik von Urteilen (der speech acts von Austin und Searle, die er als Diskurshandlungen übersetzt ${ }^{39}$ ). Die dritte ist die Ebene der Meinungen, in denen sich das Logische und das Psychologische verbinden, und mittels derer etwas vom Sprecher ausgesagt wird ${ }^{40}$ (Er greift auf Searle und Grice zurück, um die Meinung innerhalb der Kommunikation als diejenige Intention aufzuzeigen, die darin besteht, dass dass meine Intention auch wirklich als solche anerkannt wird).

Nach und nach geht Ricoeurs Reflexion von der Aussage (mit ihrem Sinn und ihrer Bedeutung) zum Ausdruck (als Handlung) über, und dann zum Ausdrückenden (als Handelndem bzw. Subjekt). Damit kann das Paradox der Kommunikation ein Stückweit näher erklärt bzw. umformuliert werden. Als Ereignis ist der Diskurs eines der Glieder in der Kette der Ereignisse, die ein Individuum bilden; aber gleichzeitig ist er auch ein Ereignis, das sich durch den darin enthaltenen Sinn von einer Einheit auf eine andere überträgt. Daher die Idee einer Dialektik zwischen dem erscheinenden und verschwindenden Ereignis und dem beständigen $\operatorname{Sinn}^{41}$. Diese Aufhebung oder Selbstüberwindung des Ereignisses innerhalb des Sinns durchdringt den ganzen Bereich der Kommunikation (Diskursivität), sowohl bei einer unmittelbaren als auch bei einer mittelbaren Kommunikation. Mit seiner Theorie des Diskurses zeigt Ricoeur, dass diese Dialektik das Fundament von Kommunikation darstellt. Anders ausgedrückt, es geschieht in der Kommunikation ein Diskursereignis, das als sinnhafte Nachricht von einer Person einer anderen Person übermittelt wird.

Diese Überlegung führt Ricoeur zu der Schlussfolgerung, den Diskurs auf folgende Weise zu definieren: ,jemand sagt jemandem etwas über etwas“42. Diese Definition verlangt nun aber nach einer Erläuterung. Erstens, wenn jemand etwas sagt, erzeugt er etwas ${ }^{43}$, und zwar einen Ausdruck, eine Diskurshandlung (Sprechakt), die eine bestimmte ,illokutionäre“ Kraft hat (d.h. also ein Befehl, ein Versprechen, eine Feststellung, usw.) ${ }^{44}$. Zweitens, wenn ,etwas

37 Diese Idee ist von Ricoeur entwickelt worden. Vgl. „Evénement et sens“, Beitrag am Institut für Philosophie, Palerme, Juni 1987, in: Raisons Pratiques 2. L'événement en perspective (J.-L. Petit hrsg.), Paris, EHESS.

38 Siehe „Discours et communication“, a. a. O., S. 56-67.

39 D.h. ,actes de discours“, und nicht wie es ins Französische übersetzt wurde, als ,actes de langage“.

40 Ricoeur spricht auch von einem „Bezug“ des Diskurses ,,auf die Gesprächteilnehmer“. Siehe „Die Metapher und das Hauptproblem der Hermeneutik“, in Vom Text zur Person, ebd., S. 114.

41 Ricoeur zufolge hatte man es schon in der Phänomenologie mit einer derartigen Dialektik zu tun, auch wenn diese genau genommen nicht sprachlich ausgerichtet war. So vertrat er die Meinung, die ein Ziel verfolgende Tathandlung könne einzig durch die identifizierbare Identität des anvisierten Sinnes erfüllt werden. Mit der Theorie des Diskurses ist diese indirekte Art und Weise symbolisch geworden. Siehe „De l'interprétation“ in Du texte à l'action, ebd., S. 30 : «En son sens rigoureux, l'intentionnalité signifie que l'acte de viser quelque chose ne s'atteint lui-même qu'à travers l'identité identifiable et ré-identifiable du sens visé - ce que Husserl appelle le «noème » ou corrélat intentionnel de la « visée noétique » »

42 Siehe z. B. „Rhétorique, poétique, herméneutique“, in Lectures 2, S. 481. In „Eine intellektuelle Autobiographie“, in Vom Text zur Person, ebd., S. 32, ist die Definition ähnlich aber genauer, d.h. ein bisschen verschieden (das wird später diskutiert - s. u.).

43 Siehe „Die Metapher und das Hauptproblem der Hermeneutik“, in Vom Text zur Person, ebd., S. 113: „Was über das Subjekt gesagt wird, ist eine Sache, was ich 'tue', indem ich spreche, ist eine andere Sache“.

44 Vgl. auch „La fonction herméneutique de la distanciation“, in Du texte à l'action, a. a. O., S. 116: „le discours renvoie à son locuteur par le moyen d'un ensemble complexe d'indicateurs, tels que les pronoms 
über etwas“ gesagt wird, ergibt sich eine feste Beziehung zwischen einem Sinn und einer Referenz: die Welt zeigt sich in der Sprache. Drittens, wie schon gesagt, wenn jemandem etwas gesagt wird, dann geht es um die Mitteilung einer Nachricht, d. h. um einen Austausch, einen Dialog. Ricoeur zufolge zeigen diese drei Vermittlungen innerhalb der Sprachphilosophie (zwischen einem Menschen und einem anderen Menschen, einem Menschen und der Welt, einem Menschen und sich selbst) dem Sprachphilosophen, dass die Sprache nicht dem Selbstzweck dient. In diesem Zusammenhang sind zwei Dinge von großer Bedeutung. Während der Diskurs als Ereignis auftaucht und dann wieder verschwindet, wird die Bedeutung im Gegensatz dazu nicht nur momentan verstanden, sondern als etwas Identisches, das immer wieder identifiziert werden kann. Dies ist die Dialektik des flüchtigen Ereignisses und des bleibenden Sinnes, also die „Überwindung“ des Ereignisses (des Sagens) in dem Sinn (dem Gesagten) (- s. u).

Im Zusammenhang mit dieser Definition scheinen folgende Anmerkungen unerlässlich. Erstens: er grenzt sich hier in drei Punkten vom Strukturalismus ab: Sprache ist nicht ihr eigenes Ziel, denn es gibt im Rahmen einer Aussage einen Handelnden, eine andere Person und eine Realität:

Die Formel, in der ich diesen Gegensatz [zum Strukturalismus] gerade zusammengefasst habe, jemand sagt jemandem etwas über etwas, eröffnete den Kampf in Wirklichkeit an drei Fronten. Außer der Wiedereinführung eines Subjekts des Diskurses im Zuge des Akts der prädikativen Synthese implizierte der Begriff des Diskurses die Anerkennung eines weiteren Sprechers als Gegenüber des Diskursaktes. Die gesamte Problematik der Intersubjektivität und der Kommunikation wurde durch das bloße Phänomen der Interlokution, das in der Definition des Diskurses enthalten ist, ins Spiel gebracht. Was die Unterscheidung zwischen Sinn und Bedeutung anbelangt, welche die Definition des Diskurses ebenfalls impliziert, so ebnete sie den Weg für eine Infragestellung eines der fundamentalen Axiome des Strukturalismus, nämlich des Verbots, auf irgend etwas Außersprachliches zurückzugreifen. (...) Sprechen, das bedeutete wieder über die Welt sprechen. (...) Das Schicksal des Subjekts war also nicht das einzige, was bei meiner Auseinandersetzung mit dem Strukturalismus auf dem Spiel stand. Die intersubjektive Dimension der Interlokution und das referentielle Anliegen der Sprache verdienten nicht weniger Aufmerksamkeit. Der Diskurs war vielmehr der Schnittpunkt dreier Problematiken: der der Vermittlung durch den objektiven Bereich der Zeichen, der die Bewusstwerdung entsprach, die jene des verletzten Cogito genannt wurde, aber auch der der Anerkennung des Anderen, die im Akt der Interlokution enthalten ist, sowie schließlich der der Problematik des Verhältnisses zur Welt und zum Sein, das der referentiellen Ausrichtung des Diskurses innewohnt. ${ }^{45}$

Mit dieser Definition vom Diskurs grenzt sich Ricoeur zum Beispiel von Foucault in Hinsicht auf das logische Problem in der Fiktion ab. Foucault hatte im Sinne der analytischen Sprachphilosophie festgestellt, dass die Aussage „Ich spreche“ zwangsläufig wahr sei, ganz im Gegensatz zur Aussage „Ich lüge“, welche die Wahrheit bedrohe ${ }^{46}$. In dieser streng performativen Wahrheit, die man als gelungene Antwort auf die Drohung von Epimenides sehen kann, erspürte er eine merkwürdige Verzweigung der Sprache. Als ob die Sprache,

personnels ; nous dirons en ce sens que l'instance de discours est sui-référentielle“.

45 Siehe „Eine intellektuelle Autobiographie“ in Vom Text zur Person, S. 34-5.

46 Foucault, La pensée du dehors, Paris, Fata Morgana, 1986, S. 10 : „Au moment où je prononce tout uniment «je parle », je ne suis menacé d'aucun de ces périls [ceux qui sont liés à la proposition «je mens »] ; et les deux propositions qui se cachent dans ce seul énoncé («je parle» et «je dis que je parle») ne se compromettent nullement. Me voilà protégé dans la forteresse indélogeable où l'affirmation s'affirme, s'ajustant exactement à elle-même, ne débordant sur aucune marge, conjurant tout danger d'erreur puisque je ne dis rien d'autre que le fait que je parle. La proposition-objet et celle qui l'énonce communiquent sans obstacle ni réticence, non seulement du côté de la parole dont il est question, mais du côté du sujet qui articule cette parole. Il est donc vrai, invinciblement vrai que je parle quand je dis que je parle“. 
genau in dem Moment, in dem sie die absolute Wahrheit ergreifen kann, plötzlich ihre wahre Natur zeigen und wieder $\mathrm{zu}$ dem Bewusstsein kommen würde, dass sie die Darstellung/Repräsentation ${ }^{47}$ verlassen könne, aus sich selbst heraustreten und in der Welt der Fiktion ohne Referenz zur „realen“ Welt ausbreiten könne. So ist es nicht wichtig, dass Foucault - in der Manier von Heidegger - in diesem „,ich spreche“ das Verschwinden des Subjekts sieht, denn die Sprache zeigt sich hier als unsicher auftauchende und wieder verschwindende Präsenz und gleichzeitig als Ursprung und Tod ${ }^{48}$. Es ist hingegen wahr, dass die Sprache in der Fiktion etwas von sich selbst zeigt, wenn auch vielleicht nicht ihr „Sein“, wie Foucault (ähnlich wie Lacan), der hierin Heidegger folgte, erklärte, sondern eine ihrer Dimensionen, und zwar ihre poetische Fähigkeit, neuen Sinn ohne direkte Referenz hervorbringen zu können. Ricoeur erkennt diese Möglichkeit der Sprache, etwas auszusagen, das nicht existiert. Doch unterstreicht er, dass der fiktive Diskurs immer ein Diskurs über etwas ist ${ }^{49}$, obwohl diese Referenz in der Textwelt (und nicht in der Welt) existiert. Seiner Meinung nach macht die Fiktion die Referenzbeziehung komplizierter, vernichtet sie aber nicht - daher seine Gedanken über die metaphorische Referenz ${ }^{50}$ (die er später als nicht ausreichend kritisierte ${ }^{51}$ ), und über die doppelte Referenz der Erzählung (fiktive und geschichtliche Diskurse benutzen gekreuzte Referenzmodi) ${ }^{52}$.

Es ist auch anzumerken, dass die pragmatische Dimension der Sprache keine sichere transzendentale Bestätigung erhält; obwohl sie als eine von einem Handelnden ausgeführte Handlung erkannt wird. Deswegen lässt Ricoeur zunächst noch eine gewisse Vorsicht bei der Einführung des Subjekts walten und wagt erst dann stärker darauf zu bestehen, als er das Argument der „Verankerung“ von Gilles-Gaston Granger übernimmt ${ }^{53}$. Man könnte hervorheben, dass andere Autoren im Vergleich dazu diese transzendentale Dimension der Pragmatik nicht so klar erkannt haben. Zum Beispiel funktioniert der Begriff der „Aussage“ („énoncé") bei Foucault, in L'Archéologie du savoir, in einer merkwürdigen Art und Weise, die fast nur in einer Semantik ohne Pragmatik besteht ${ }^{54}$ - im Strukturalismus (bei Barthes oder Derrida) wird der Autor im Allgemeinen nicht als Handelnder anerkannt, ganz so als sei die Sprache (oder der Text) nur ein auto-poetisches Zeichensystem ohne Autor ${ }^{55}$. Auf ähnliche Weise versucht auch Davidson, die Pragmatik, d.h. die Indexikalität und die illokutionäre Kraft, in die Semantik zu integrieren ${ }^{56}$. In Anbetracht dessen, dass die Semantik durch eine

47 Bei Foucault ist immer der „Diskurs“ mit „Repräsentation“ verbunden.

48 Foucault, „La pensée du dehors“, ebd., S. 13: die Aussage „Ich spreche“ würde im Gegensatz zu dem kartesianischen „Ich denke“ nicht als eine Referenz und ein Inneres, sondern als eine Loslösung von der Referenz und eine Eroberung des Äußeren funktionieren. Daher auch die Idee von einer „Pensée du dehors“.

49 Siehe „Die Metapher und das Hauptproblem der Hermeneutik“, in Vom Text zur Person, ebd., S. 112-113: „Der Diskurs beinhaltet (...) die Polarität von Sinn und Referenz, das heißt, die Möglichkeit, zwischen dem, was durch den Satz als Ganzes und durch die Wörter als Teile des Satzes gesagt wird, und dem, worüber etwas gesagt wird, zu unterscheiden. Sprechen, das heißt, etwas über etwas sagen“.

50 Siehe Die lebendige Metapher, München, Fink, 1986, vorletzte Abhandlung (vii: „Metapher und Referenz").

51 Vgl. „Eine intellektuelle Autobiographie“, in Vom Text zur Person, ebd., S. $46-48$ (Ricoeur erklärt, dass in dieser Reflexion die Betonung der Rolle des Lesens fehlte).

52 Siehe Zeit und Erzählung 3/ Die erzählte Zeit, ebd., zweiter Teil.

53 Vgl. Das Selbst als ein Anderer, Wilhelm Fink Verlag, München, 1996, S. 63-8. Zu diesem Argument der Verankerung und zur ,reinen Pragmatik“, betrachtet von einem semiotischen und transzendentalen Standpunkt,, siehe meinen im Jahr 2004 veröffentlichten Artikel „Granger et la Critique de la Raison Symbolique“: http://www.revue-texto.net/Inedits/Lacour_Granger.html.

54 Michel Foucault, Archäologie des Wissens, Frankfurt, Suhrkamp, 1988.

55 Foucault schrieb auch über den „Tod des Autors“. Vgl. „Qu'est-ce qu'un auteur?“, Bulletin de la Société française de philosophie, ${ }^{\circ} 3$, Juli-Sept. 1969; vgl. auch Dits et Ecrits I, Paris, Gallimard (Quarto), S. 817 ff.

56 Siehe Davidson, Inquiries into Truth and Interpretation, Oxford University Press, 1984. Davidson erkennt zwar die pragmatische Ebene der Sprache an, betrachtet ihre Rolle aber als sekundär. Einen solchen 
extensionale Logik formalisiert wird und sich die (so genannte „radikale“) Interpretation des Sinnes auf den Bereich der Psychologie beschränkt, kann die Bedeutung der Sprache als invariant angesehen werden. Die Pragmatik aber ist ein Faktor der Mehrdeutigkeit der Sprache (z. B. spielt die Indexikalität bei der Bedeutung des Kontextes eine große Rolle). Damit sein Versuch gelingt, braucht Davidson aber mindestens eine „modale“ Semantik ${ }^{57}$, durch die jedoch auch nur eine reduzierte Pragmatik berücksichtigt wird ${ }^{58}$. In der zeitgenössischen Philosophie von Vincent Descombes wiederum wird, auf Kosten der Pragmatik, die nicht wirklich als das Zeichen der Handlung eines Handelnden angesehen wird, das Gewicht auf die Syntax gelegt ${ }^{59}$, im Unterschied zu der bevorzugten Stellung, die die Phonologie im französischen Strukturalismus einnimmt ${ }^{60}$.

Das Wichtigste an dieser Definition des Diskurses von Ricoeur ist demnach die Dialektik des Ereignisses und der Bedeutung. Als Ereignis geschieht der Diskurs, d.h. er wird als Handlung von einem Handelnden ausgeführt. Aber als Bedeutung überlebt er diesen Augenblick und beginnt seinen besonderen Weg. Ganz offensichtlich und lesbar ist diese Dialektik im Text, in dem es um vermittelte Kommunikation, und nicht mehr um einen Dialog geht. Dass diese Dialektik aber auch im Gespräch existiert, sozusagen sobald die Diskursivität beginnt, versucht Ricoeur mit einer Untersuchung des Begriffs Werk (s.u.) nachzuweisen. Dabei spricht Ricoeur von einem Textwerden des Diskurses vom Dialog ausgehend $^{61}$.

Genauso wichtig jedoch ist die Umkehrung, durch die Ricoeur die Sprachwissenschaft vom Virtuellen zum Aktuellen zurückbringt. In der strukturalistischen Linguistik ignorierten die Nachfolger von Saussure (wegen der Überlegenheit der „langue“ gegenüber der ,parole“), sowie die Schule von Chomsky (bei der die Kompetenz die Performanz überwiegt), die tatsächlichen sprachlichen Objekte, d. h. die aktuellen Diskurse. Daher das immer größere Interesse der Linguistik heutzutage an den Korpora ${ }^{62}$, die tatsächlich stattgefundene sprachliche Produktionen dokumentieren.

Gegen diese Auslegung des Diskursbegriffes kann man einwenden, dass Ricoeur in dem Text „Discours et communication“ die Kommunikation vom Standpunkt der transzendentalen Phänomenologie aus betrachtet. Es geht dabei nämlich um die Möglichkeit für das transzendentale Bewusstsein, eine Lücke oder eine Distanz $\mathrm{zu}$ überbrücken, um das Bewusstsein des Anderen zu erreichen, also darum, dass eine Monade mit einer anderen

„residualen“ Wert der Pragmatik kritisiert K. O. Apel bei Tugendhat (Vorlesung zur Einführung in die Sprachanalytische Philosophie, Suhrkamp 1976) in seinem Artikeln: „Die Logosauszeichnung der menschlichen Sprache. Die philosophische Relevanz der Sprechakttheorie“, in: H.-G. Boßhardt (Hrsg.), Perspektiven auf Sprache, Berlin / New York, S. 45-87, 1986. Über diesen ,pragmatische[n] Paralogismus“, vgl. auch Transformation der Philosophie, II, Suhrkamp, 1973, S. 406 ff.

57 Pascal Engel hob dieses Argument hervor. Siehe Davidson et la philosophie du langage, Paris, PUF, 1994 ; Kapitel 3.

58 Ähnlich äußert sich auch Gilles-Gaston Granger: siehe Langages et épistémologie, Paris, Klincksieck, 1979, Kapitel 4: „L'expression des formes logiques dans la langue naturelle“, S. 69-94. Z. B. in den Zeitlogiken, wie bei Prior, wird die Pragmatik begrenzt. Der Grund dafür liegt in dem Unterschied zwischen dem Logischen und dem Proto-logischen (siehe http://www.revue-texto.net/Inedits/ Lacour_Granger.html, 3.5).

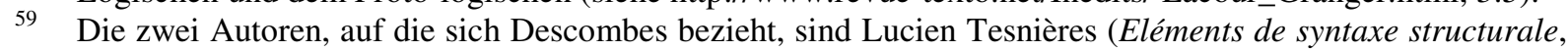
Paris, Klincksieck, 1969) und Anthony Kenny (Action, Emotion and the Will, London, Routledge, 1963). Vgl. zum Beispiel Vincent Descombes, Le complément de sujet. Enquête sur le fait d'agir de soi-même, Paris, Gallimard, 2004.

60 Siehe Le complément de sujet, ebd., Kapitel XV und XVI, S. 133-156.

61 Siehe „De l'interprétation““, in Du texte à l'action, S. 35: „....) l'écrit s'arrache aux limites du dialogue face à face et devient la condition du devenir-texte du discours“. Versuch einer Übersetzung: „,das Geschriebene hebt sich über die Grenzen des Dialogs im Gegenüber und wird zu der Bedingung des Textwerdens des Diskurses“.

62 Siehe zum Beispiel Damon Mayaffre, "Rôle et place des corpus en linguistique"; http://www.revuetexto.net/Corpus/Publications/Mayaffre_Corpus.html. 
Monade in Berührung kommt ${ }^{63}$. Meiner Meinung nach bedient sich Ricoeur in diesem Text der transzendentale Philosophie aber nur, um Jakobsons allgemeine Definition der Kommunikation in Frage zu stellen, in einer völlig klassischen „kritischen“ philosophischen Geste, die sich auf den sokratischen ,thaumaston“ zurückführen lässt. Ricoeur versucht lediglich, die Kommunikation als einen Prozess zu begreifen, der sich nicht von selbst versteht und bringt den transzendentalen Aspekt nur zu dem Zwecke ins Spiel, um die banale Tatsache der Kommunikation durch ein philosophisches Paradox zu „dramatisieren“. Hinter der Rhetorik von einer Hinwendung von dem natürlichen zum phänomenologischen Zustand verbirgt sich in Wahrheit der Versuch, im Diskurs die Möglichkeitsbedingungen der Kommunikation zu finden ${ }^{64}$.

\section{Die Geschichtlichkeit des Diskurses : vom Dialog zum Text}

Es ist sehr wichtig die Diskursivität als eine Gesamtheit zu verstehen, die aus verschiedenen Teilen, vom Ausdruck des Diskurses bis hin zum weiteren Sinnleben des Textes, besteht. In der konkreten Absicht, die Beziehung des Textes zum Dialog zu beleuchten, fügt Ricoeur in die Diskursivität, zwischen den Ausdruck der Sprache als Diskurs einerseits und die Dialektik von Sprache und Schrift andererseits die Idee der Äußerung des Diskurses als strukturiertes (aufgebautes) Werk ein.

\subsection{Was ist ein Werk?}

Die sprachliche Äußerung in Form des Diskurses gibt dem Sinn eine wichtige Bedeutung, welche alle (lokutionären, illokutionären, perlokutionären) Aspekte und Ebenen des intentionalen Ausdrucks in sich vereint, die wiederum ihrerseits den Ausdruck des Diskurses im Werk und in der Schrift ermöglichen. Insbesondere im Werk wird der Sinn noch erweitert.

Das Werk besteht in der Äußerung des Diskurses, sozusagen in statu nascendi. Es ist quasi die erste Etappe der Äußerung, die erste objektivierbare Phase der Dialektik des Ereignisses und des Sinnes und somit das erste beobachtbare Moment der Distanzierung in der Kommunikation, durch die das „Gesagte“ dem „Sagen“ entgeht und der Sinn seinen eigenen Weg geht. Bei dem diskursiven Werk handelt es sich um die Dekontextualisierung des Sinnes in seinem Anfangsstadium (inchoativen Stadium), um das clinamen des Sinns.

Ricoeur zufolge zeichnet sich das Werk durch drei Aspekte aus ${ }^{65}$. Erstens handelt es sich hierbei um eine Sequenz, die länger als der Satz ist. Und diese ,große Sinneinheit“ ruft ein neues Verständnisproblem hervor, welches das gesamte Werks betrifft - und später noch zu behandeln sein wird - (s. u.). Zweitens fügt sich das Werk einer Kodifizierungsform, die sich auf die Komposition auswirkt: so wird der Diskurs Erzählung, Gedicht, Essay usw. (man nennt diese Kodifizierung die literarische „Gattung“). Schließlich erfährt ein Werk eine einzigartige Konfiguration, die sie mit einem Individuum gleichstellt und als „Stil“ bezeichnet wird.

In Bezug auf „das Werk“ muss noch eine weitere Präzisierung erfolgen: Komposition, Gattung und individueller Stil sind, wie Ricoeur betont, Kategorien der Produktion und der Arbeit, durch die die Sprache als ein geschaffenes Material eingestuft wird. Damit kann der Diskurs als Objekt einer praxis und einer technè betrachtet werden. Diesen Gedanken

63 Siehe „Discours et communication“, ebd., S. 52-54, in dem Husserl mit Leibniz in Verbindung gebracht wird. Ricoeur sagt, dass es sein Ziel sei, den Diskurs als Überwindung (Übertretung) einer monadischen Nicht-Kommunizierbarkeit $\mathrm{zu}$ verstehen (,comprendre le discours comme transgression de l'incommunicabilité monadique“) (ebd., S. 53).

64 Daher die Nähe von Ricoeur und Granger, wobei letzterer diese Bedingungen in einer symbolischen Philosophie sucht.

65 Siehe „La fonction herméneutique de la distanciation“, in Du texte à l’action, a. a. O., S. 120-124. 
übernimmt Ricoeur von Gilles-Gaston Granger und dessen Versuch einer Philosophie des Stils $(1968)^{66}$. Am wichtigsten sind für Ricoeur aber folgende Aspekte: dass im Werk der Sinn eine besondere (kompliziertere) Dimension erhält, und dass das Werk eine praktische Vermittlung zwischen der Irrationalität des Ereignisses und der Rationalität des Sinns ist. Tatsächlich geht es bei dem Diskurs um eine strukturierte sprachliche Produktion, die in Beziehung zu einer konkreten und komplexen - offenen - Situation (mit Tendenzen und Konflikten) steht. Ein Werk als Ereignis zu betrachten, heißt für Ricoeur die Beziehung zwischen der Situation und dem Projekt im Prozess der Restrukturierung zu verstehen. Der Stil ist zur gleichen Zeit Ereignis und Sinn; er verbessert die Lesbarkeit eines Werks, dessen Einzigartigkeit den ereignishaften Charakter des Diskurses illustriert.

Der Begriff des Subjekts im Diskurs wird ein anderer, wenn der Diskurs ein Werk wird. Genau wie der Stempel eines Möbelstücks die Arbeit des Handwerkers auszeichnet, scheint der Begriff des „Autors“, der das sprechende Subjekt bezeichnet, das Korrelat der Individualität des Werks zu sein. Weil der Stil eine Arbeit ist, die etwas Individuelles produziert, bezeichnet er wiederum rückwirkend seinen Autor. Deswegen gehört der Begriff des Autors zur Stilistik.

Am wichtigsten im Begriff des Werkes ist die Einführung einer Idee der Komposition. Als „einzigartiges Ganzes“ erschöpft sich das literarische Werk „nicht in einer Folge von Sätzen, die alle für sich selbst verständlich wären, es ist ein Gefüge von Themen und Äußerungen, das auf unterschiedliche Weise konstruiert werden kann ${ }^{\text {“67 }}$. In einer derartigen Gesamtheit ist das Verhältnis zwischen dem Teil und dem Ganzen zirkulär: „Die Voraussetzung eines gewissen Ganzen geht der Wahrnehmung einer bestimmten Anordnung der Teile voran, und indem man die Details errichtet, baut man das Ganze auf "68. Wegen dieser Einzigartigkeit ist ein Text „eine Art Individuum wie ein Lebewesen oder ein Kunstwerk“, deren Verständnis von der reflexiven Urteilskraft abhängt ${ }^{69}$.

Unter dem Eindruck der Objektwerdung des Diskurses in einem Werk und dem Erfolg (nach Ricoeur) der strukturalistischen Analysemethoden entwickelt sich der von Dilthey übernommene Widerspruch zwischen dem Erklären und dem Verstehen ${ }^{70}$ in eine Dialektik, die beide zusammenbringt ${ }^{71}$. Deswegen wird die Erklärung als der notwendige Weg zum Verstehen betrachtet.

Ricoeur irrt sich jedoch, wenn er meint, dass die Grundhypothese jeder strukturalistischen Textanalyse, dass nämlich ,die großen Einheiten der Sprache, das heißt die dem Satz übergeordneten Einheiten Strukturen zeigen, die mit denen der kleinen Einheiten der Sprache vergleichbar sind“, „,öllig legitim“ sei $^{72}$. Sein erster Irrtum besteht darin, dass, was er

66 Gilles-Gaston Granger, Essai d'une philosophie du style, Paris, Aubier, 1968. Granger versucht, eine Erkenntnis des Individuellen in einer rationalen Philosophie zu schaffen. Z.B. versucht er, hierin Kant (in der dritten Kritik) widersprechend, zu argumentieren, dass ein Kunstwerk nicht nur eine Universalität ohne Begriff ist, sondern auch eine begriffliche Individualität (ebd., S. 7). Dieses „,klinische“ Problem (Erkenntnis des Individuellen) wird durch formales Denken durchgeführt - siehe z. B. Formal Thought and the Sciences of Man, Dordrecht, Boston, D. Reidel Pub. Co., Hingham (MA), 1983.

67 Siehe „Die Metapher und das Hauptproblem der Hermeneutik“, in Vom Text zur Person, ebd., S. 124.

68 Vgl. Ricoeur, a. a. O., S. 124.

69 Siehe Ricoeur, ebd., S. 124. Ricoeur zitiert ausdrücklich die dritte Kritik Kants.

70 Zu diesem Widerspruch, siehe „Was ist ein Text?“, in Vom Text zur Person, ebd., S. 86-90. Wie die französische Übersetzerin (Sylvie Mesure, Verlag Cerf, 1988) feststellt, wurde dieser Widerspruch aber in Der Aufbau der geschichtlichen Welt in den Geisteswissenschaften (Gesammelte Schriften Bd. VII. 5. Aufl. Stuttgart, Göttingen 1968) reduziert. Nach der Meinung Heinz Wismanns wurde Ricoeur von der Interpretation Gadamers beeinflusst, der stets die psychologische Art und Weise der Hermeneutik Diltheys kritisiert (siehe Johann Michel, Paul Ricoeur, une philosophie de l'agir humain, Paris, Cerf, S. 147-8). Das hat Ricoeur später anerkannt.

71 Siehe „Was ist ein Text?“, in Vom Text zur Person, a. a. O., S. 90-97.

72 Siehe: „Was ist ein Text?“, Vom Text zur Person, a. a. O., S. 92. Ricoeur spricht auch von einer 
„struktural“ nennt, in Wahrheit keine richtige (d. h. formale, mathematische) Struktur ist, wie auch Gilles-Gaston Granger bemerkte ${ }^{73}$, sondern einfach eine besondere Regelmäßigkeit des impliziten semantischen Aufbaus des Textes, die nur teilweise formalisiert werden kann ${ }^{74}$. So handelt es sich bei dieser Regelmäßigkeit nicht um ein „Strukturgesetz“, wie Levi-Strauss dachte, auch wenn sie etwas mit der Erklärung zu tun hat ${ }^{75}$. Ricoeurs zweiter Irrtum ist eine Konsequenz des erstens: bei dieser impliziten Regelmäßigkeit des Textaufbaus geht es um eine semantische Untersuchung, die, in ihrer Eigenschaft als „textuelle“ keine „strukturelle“ sondern eine „,interpretative“ ist, wie François Rastier im Gegensatz zu Greimas (und anderen) unterstrich ${ }^{76}$. Daher liegt die Dialektik zwischen dem Erklären und dem Verstehen, die allerdings meiner Meinung nach beibehalten werden sollte, auch nicht wirklich dort, wo Ricoeur sie sah. Denn eigentlich war das, was er „strukturale Erklärung“ nannte, schon teilweise eine Interpretation. Man sollte also eher von einer Dialektik zwischen der Formalisierung und der semantischen Interpretation sprechen ${ }^{77}$.

\subsection{Die Schriftlichkeit als Distanzierung vom Gespräch}

Die Schrift scheint nur ein materielle Fixierung der Sprache zu sein, welches das Ereignis des Diskurses vor der Zerstörung bewahrt ${ }^{78}$. Ricoeur weist jedoch darauf hin, dass diese Fixierung, ein viel erheblicheres Problem sichtbar macht: das Problem der Distanzierung, das alle Aspekte des Diskurses betrifft.

Zunächst wird der Text unabhängig von der Autorenabsicht betrachtet - wie schon Platon (im Phaidros) mit der berühmten Metapher vom Text als „Waisenkind“ vorgab. Das, was der Text bedeutet, fällt nicht mehr mit der Meinung des Autors zusammen. Eine derartige Trennung von logischer und psychologischer Bedeutung stellt eine Verfremdung dar, die bei Ricoeur nicht nur eine negative Dimension hat, wie etwa bei Gadamer, sondern gleichzeitig auch eine positive und produktive Kraft. Dieses Phänomen erklärt Ricoeur sehr gut:

„Übertragung der Methode der Kommutation von der Phonologischen Ebene auf die Ebene der Einheiten der Erzählung“" (vgl. ebd., S. 96).

73 Siehe sein Vorwort zur zweiten Ausgabe von Pensée formelle et sciences de l'homme, (Paris Aubier, 1960) 1968 : „Au lecteur, sur les structuralismes“.

74 Darauf besteht Granger in seiner „Kritik der Symbolischen Vernunft“ (siehe seine erneute Version der „transzendentalen Dialektik“ : http://www.revue-texto.net/Inedits/ Lacour_Granger.html, 4).

75 Diese Erklärung ist aber weder strukturell noch „gesetzlich“, sondern kasuistisch und beschreibend. Meine Hypothese, die ich von Clifford Geertz und Granger übernehme, lautet daher, dass bei Levi-Strauss eine Radikalisierung des komparativen Denkens stattfindet (z.B. im Vergleich zu einem anderen Mythologen, der Foucault auch sehr beeinflusst hat: Georges Dumezil). Über diese kritisierte Idee einer kasuistischen Methode in den Kulturwissenschaften, vgl. Meine Buchrezension von Pensée par cas (JC Passeron, J. Revel hrsg.), Paris, EHESS, 2004: „Thinking by Cases, or: how to Put Social Sciences back the Right Way Up“, http://www.espacestemps.net/document1395.html.

76 Siehe zum Beispiel Carine Duteil-Mougel, „Introduction à la sémantique interprétative“, http://www.revuetexto.net/Reperes/Themes/Duteil/Duteil_Intro.html

77 Das ist genau das, was François Rastier meint, wenn er von einer, durch Computer gestützten Interpretation“, oder auch einer „,numerischen Philologie“ spricht (in der Korpuslinguistik).

78 Vgl. „La fonction herméneutique de la distanciation“, in Du texte à l'action, ebd., S. 124 f. ; und auch: „Was ist ein Text?“, in Vom Text zur Person, a. a. O., S. 80: ,(...) die Überzeugung, dass die Schrift ein fixiertes Sprechen ist, dass die Inskription - sei es als Schrift oder Aufzeichnung - eine Inskription von Sprechen ist, eine Inskription, die dem Sprechen dank des substantiellen Charakters des Eingravierens seine Dauerhaftigkeit gewährleistet“. Ganz im Gegenteil dazu fragt sich Ricoeur „ob der Text nicht wirklich Text ist, wenn er sich nicht darauf beschränkt, ein vorheriges Sprechen zu transkribieren, sondern wenn er unmittelbar in das Schriftzeichen inskribiert, was der Diskurs sagen will“ (vgl. ebd., S. 81). Ein Argument dafür findet er in dem Unterschied zwischen dem Gespräch (Dialog) und dem Lesen. Deswegen ist die Schrift kein diskursiver Ersatz, sondern, neben dem Sprechen, eine echte Art von Diskurs: „(...) das, was zum schriftlichen Ausdruck gelangt, der Diskurs als Absicht ist, etwas zu sagen, und dass die Schrift eine unmittelbare Inskription dieser Intention ist“ (vgl. ebd., S. 82). 
Man glaubt zu wissen, was der Autor eines Textes ist, weil man seinen Begriff von dem des Sprechers der gesprochenen Äußerung ableitet. Das Subjekt des Sprechens, sagt Benveniste, ist dasjenige, das sich selbst bezeichnet, indem es „ich“ sagt. Wenn der Text die Stelle des Sprechens einnimmt, gibt es genau genommen keinen Sprecher mehr, zumindest nicht im Sinne einer unmittelbaren und direkten Selbstbezeichnung dessen, der in der Diskursinstanz spricht. An die Stelle dieser Nähe des sprechenden Subjekts zu seinem eigenen Sprechen tritt eine komplexe Beziehung des Autors zum Text, die zu sagen erlaubt, dass der Autor durch den Text eingesetzt wird, dass er sich selbst im Raum der durch die Schrift entworfenen und inskribierten Bedeutung aufhält. Der Text ist der Ort selbst, an dem der Autor ankommt ${ }^{79}$.

Diese Distanzierung führt sodann auch zur Unabhängigkeit des Diskurses von dem sozial verankerten Ort seiner Produktion. Daher die Idee einer allgemeinen Dekontextualisierung des Textes, sowohl von seinen psychologischen, als auch seinen soziologischen Bedingungen, ganz so als ob dieser Diskurs ohne pragmatische Ebene wäre (es handelt sich dann um eine Pragmatik, die nicht direkt, sondern mittelbar ist).

Zum Ausgleich erlaubt diese Dekontextualisierung dann weitere Rekontextualisierungen (pragmatische Wiederverankerungen), und zwar mithilfe verschiedener Lektüren des Textes $^{80}$. Jeder kann den Text lesen. Auf dieser Unabhängigkeit von der Gesprächssituation besteht Ricoeur umso stärker, als das Lesen in der romantischen Tradition der Hermeneutik (von Schleiermacher bis Dilthey) eine Arte von Dialog ist ${ }^{81}$ :

Das Verhältnis von Schreiben und Lesen ist nämlich kein besonderer Fall des Verhältnisses von Sprechen und Antworten. Es ist kein Verhältnis des Gesprächs, es ist kein Fall eines Dialogs. Es genügt nicht zu sagen, dass die Lektüre ein Dialog mit dem Autor durch sein Werk hindurch ist. Man muss feststellen, dass das Verhältnis des Lesers zum Buch von ganz anderer Art ist. Der Dialog ist ein Austausch von Fragen und Antworten. Es gibt keinen derartigen Austausch zwischen dem Schriftsteller und dem Leser. Der Schriftsteller antwortet dem Leser nicht. Vielmehr trennt das Buch den Akt des Schreibens und den Akt des Lesens in zwei Vorgänge, die nicht miteinander in Verbindung stehen. Der Leser ist beim Schreiben abwesend, der Schriftsteller ist beim Lesen abwesend. Der Text bewirkt so eine doppelte Ausblendung des Lesers und des Schriftstellers. Auf diese Weise tritt er an die Stelle der dialogischen Beziehung, welche die Stimme des einen unmittelbar mit dem Gehör des anderen verbindet ${ }^{82}$.

Es steht für Ricoeur fest, dass diese Distanzierung eine logische Dimension, d. h. ein Aspekt des Diskurses selbst ist und nicht das Produkt einer Entscheidung für eine Methode wie bei Gadamer. Deswegen wird die Distanzierung nicht als etwas Überflüssiges betrachtet, sondern als etwas Konstitutives des Textes, der ein diskursives Produkt neben dem Sprechen ist und dessen Einzigartigkeit anerkannt werden muss. Deshalb ist die Distanzierung nicht nur ein Hindernis für das Verstehen, sondern gleichzeitig auch seine Bedingung. Daraus folgt eine komplementäre Beziehung zwischen Objektivierung und Interpretation, die keineswegs ein Widerspruch im Sinne der romantischen Tradition der Hermeneutik ${ }^{83}$ ist.

Hier kann man anmerken, dass für Ricoeur der Text, der jenseits der Dialogsituation ein offenes, schweifendes „Schicksal des Sinnes erlaubt“, bezeichnend für die allgemeine

79 Siehe „Was ist ein Text?“, in Vom Text zur Person, ebd., S. 85.

80 Vgl. „La fonction herméneutique de la distanciation“, in Du Texte à l'action, a. a. O., S. 125.

81 Erstaunlicherweise stimmt auch ein Autor der analytischen Philosophie wie Davidson damit überein. Siehe z. B. „Locating Literary Language“ in Truth, Language and History, New York, Oxford University Press, 2005.

82 Siehe „Was ist ein Text?“, in Vom Text zur Person, ebd., S. 81. Zur gleichen Zeit ändert sich die traditionelle hermeneutische Definition der Aneignung (oder Anwendung), die kein zeitgenössisches, sondern ein distanziertes Verstehen ist (siehe: „La fonction herméneutique de la distanciation“, in Du texte à l'action, ebd., S. 130-1).

83 Siehe : "La fonction herméneutique de la distanciation", in Du texte à l'action, ebd., S. 125. 
diskursive Dialektik des Ereignisses und der Bedeutung ist. Das bedeutet: nur indem sie die Einzigartigkeit der Frage des Texts anerkennt, kann diese Dialektik in allen Diskursvarianten, so auch im Gespräch ${ }^{84}$, entdeckt werden; hieraus erklärt sich auch die Wichtigkeit des Werkes als erste Etappe der Distanzierung. Man könnte sagen, dass Ricoeur, im Gegensatz zur romantischen Tradition, die das Lesen als Dialog versteht, auf dem besteht, was schon im Dialog hermeneutisch anklingt: dass nur eine gemeinsame Theorie des Diskurses Dialog und Text zusammenbringen und charakterisieren kann. Diese allgemeine Theorie des Diskurses erlaubt es Ricoeur auch, Derridas Theorie der Schriftlichkeit zu kritisieren, der dieser Eigenschaften zuschreibt, die Ricoeur zufolge zum Diskurs im Allgemeinen gehören ${ }^{85}$.

\subsection{Die Welt des Textes}

Ein wesentlicher Unterschied zwischen den diskursiven Gattungen Dialog und Text ist die Veränderung des Referenzproblems ${ }^{86}$. Während beim Austausch der gesprochenen Äußerung der eine Sprecher für den anderen anwesend ist (und auch ,die Situation, die Stimmung, das Milieu des Diskurses“, durch die Verankerungssymbole der Sprache (die deiktischen oder ostensiven Indexwörter), die die Diskursinstanz zeigen), verschwindet diese direkte pragmatische Beziehung im Text: „So beugt sich im lebendigen Sprechen der ideale Sinn dessen, was man sagt, zur realen Referenz zurück, nämlich zu dem, worüber man spricht. Im äußersten Fall neigt diese wirkliche Referenz dazu, mit einer ostentativen Bezeichnung verwechselt zu werden, bei der das Sprechen wieder bei der zeigenden, hinweisenden Geste ankommt. Der Sinn liegt in der Referenz zugrunde, und diese im Zeigen“"87. Deswegen spricht man von der Umwelt der Sprachlichkeit ${ }^{88}$. Beim Text handelt es sich um das Gegenteil einer vorübergehenden Neutralisierung der direkten Referenz, d. h. nicht um eine Vernichtung, sondern um eine Komplizierung: „Die Bewegung der Referenz zum Zeigen hin wird zur gleichen Zeit aufgehalten, zu welcher der Dialog durch den Text unterbrochen wird. Ich sage freilich aufgehalten und nicht unterlassen. (...) Der Text (...) ist nicht ohne Referenz. Es wird genau die Aufgabe der Lektüre als Interpretation sein, die Referenz zu vollziehen. Zumindest in dieser Schwebe, in der die Referenz aufgeschoben ist, befindet sich der Text gewissermaßen ,in der Luft“, außerhalb der Welt oder ohne Welt. Zugunsten dieser Entwertung des Bezugs zur Welt ist jeder Text frei, in ein Verhältnis zu allen anderen Texten

84 Vgl. ebd., S. 124: die Dialektik der Sprachlichkeit und der Schriftlichkeit ist auf einer anderen primitiveren Distanzierungsdialektik aufgebaut, welche zum sprachlichen Diskurs gehört, in dem dieser Diskurs ist; „deswegen soll in dem Diskurs selbst die Entstehung aller anderen Dialektiken entdeckt werden“ (,dialectique de distanciation (...) qui appartient au discours oral en tant qu'il est discours; c'est donc dans le discours même qu'il faut chercher la racine de toutes les dialectiques ultérieures“.

85 Daher die Verworrenheit zwischen dem, was semiologisch, d. h. nur virtuell ist, und dem, was diskursiv, d. h. effektiv/aktuell ist : „,...) vous êtes obligé de surcharger la théorie de l'écriture de tout ce qui n'a pas été fait en son lieu propre, qui est une théorie du discours. Si cette théorie du discours est faite, elle peut rendre compte des caractères d'écriture que vous avez montrés parce que c'est dans la discursivité elle-même qu'il y a tous ces traits que vous avez attribués à l'écriture. C'est un peu sur ce problème du discours que, pour ma part, j'aimerais discuter avec vous“ (Auseinandersetzung im Symposium Montreal, 1971 ; Paul Ricoeur, a. a. O., S. 22). Vgl. auch S. 23 seine Kritik der Differenz: „Il y a des différences entre des signes, puis il y a le fait que le sujet n'est pas le prédicat, enfin il y a partout des différences ; mais ce qui est important c'est que le discours produit, par des différences propres qui ne sont pas des différences sémiologiques, des effets de discours qui ne sont pas des effets de signes“.

86 Ricoeur spricht von der „Umwälzung, welche die referentielle Beziehung der Sprache zur Welt berührt, wenn der Text die Stelle des Sprechens einnimmt“" „Was ist ein Text?“, in Vom Text zur Person, a. a. O., S. 3).

87 Vgl. Ricoeur, ebd., S. 83-4.

88 Siehe: "Le modèle du texte : l'action sensée considérée comme un texte", in Du texte à l'action, ebd., S. 210. Ricoeur spricht von einer Erweiterung der Umwelt zur Welt mit der textuellen (distanzierten) Dimension des Diskurses. 
zu treten, die gerade den Platz der vom lebendigen Sprechen gezeigten Realität der Umstände einnehmen“689.

Mit diesem Begriff der Welt des Textes trennt sich Ricoeur vom Strukturalismus und von der romantischen Hermeneutik, wobei das Programm seiner Hermeneutik nicht darin besteht, eine Struktur oder ein Genie zu entdecken, sondern eine Welt zu analysieren ${ }^{90}$. Dass er den Begriff des Verstehens von Heidegger übernimmt, erklärt seine Definition der Interpretation als die Auslegung des in-der-Welt-Seins des Textes - dieses in-der-Welt-Sein breitet sich vor dem Text aus ${ }^{91}$. Seine Überlegungen zu den Überkreuzungen und Unterschieden zwischen der Referenz in der Fiktion und in der Historiographie - bzw. die Vermittlungen der Referenz durch die so genannten ,überkreuzten“ Referenzmodi - hat Ricoeur in Zeit und Erzählung, Band $3^{92}$, festgehalten.

Von erheblicher Wichtigkeit ist außerdem sein Verständnis von einer Absicht des Textes selbst, die nicht mehr nur eine ,subjektive Operation der Interpretation als auf den Text gerichteter Akt“ ist, sondern vielmehr ,eine objektive Operation der Interpretation (...), welche der Akt des Textes wäre“" ${ }^{\text {"93 }}$. Damit wird ein neuer Begriff der Interpretation ${ }^{94}$ entwickelt, der nicht mehr psychologisch, sondern logisch ist ${ }^{95}$.

Ricoeur besteht auf dem aktuellen Aspekt der Textinterpretation, die als Aneignung betrachtet werden soll. Durch das Lesen wird der Text „aktualisiert“: „Die Lektüre ist wie eine Ausführung einer musikalischen Partitur, sie markiert die Verwirklichung, die Realisierung der semantischen Möglichkeiten des Textes“"96. Mit dieser Aktualisierung wird ein entscheidender Aspekt der Lektüre erkannt: „nämlich, dass sie den Diskurs des Textes in einer Dimension vollendet, die jener des Sprechens ähnlich ist ${ }^{\star 97}$. Mit dieser Bemerkung unterstreicht Ricoeur, dass die Kriterien der Diskursivität nicht die der Aussprache sind, sondern die eines sprachlichen Ereignisses ${ }^{98}$. Weil die Sätze des Textes hic et nunc bedeuten, bezieht sich der ,,aktualisierte“ Text auf eine Umgebung und ein Publikum. Damit nimmt er „seine - zurückgehaltene und schwebende - Bewegung der Referenz auf eine Welt und auf Subjekte wieder auf“99. Diese Interpretation, obwohl sie kein Sprechen ist ${ }^{100}$, gehört zur Diskursivität: ,die Lektüre kommt konkret in einem Akt zum Abschluss, der für den Text ist, was das Sprechen für die Sprache ist: nämlich Ereignis und Diskursinstanz “" ${ }^{101}$.

Mit diesem neuen Begriff der Interpretation nähert sich Ricoeur und trennt sich zugleich von dem Begriff hermeneia von Aristoteles und der semiosis von Peirce ${ }^{102}$. Die hermeneia

89 „Was ist ein Text“, in Vom Text zur Person, ebd., S. 84. Daher, nach Ricoeur, die Möglichkeit der Ausblendung der realen Welt einer Literatur, als Quasi-Welt der Texte und eine Art „Aura“, welche die Werke entfalten, wie in der ,griechischen Welt“. Diese Quasi-Welt darf imaginär genannt werden, indem „,sie genau an der Stelle durch die Schrift vergegenwärtigt wird, an der sie durch die gesprochene Äußerung gezeigt wurde“, obwohl dieses Imaginäre selbst „eine Schöpfung der Literatur“ ist (vgl. ebd., S. 84-5).

90 Siehe: "La fonction herméneutique de la distanciation", in Du texte à l'action, ebd., S. 126.

91 Ebenda, S. 128: „interpréter, c'est expliciter la sorte d'être-au-monde déployé devant le texte“.

92 Siehe auch „Die Verbindung von Fiktion und Geschichte in der Refiguration der Zeit“, in „Die erzählte Zeit“, Vom Text zur Person, a. a. O., S. 199 sq.

93 Vgl.: „Was ist ein Text?“, in Vom Text zur Person, ebd., S. 104.

94 Ebenda, S. 98 (Titel).

95 Diesen Versuch bemerkt Ricoeur auch bei Dilthey. Vgl. Ricoeur, a. a. O., S. 88.

96 Ebd., S. 100

97 A. a. O.

98 Ebenda: „Was hier vom Begriff der sprachlichen Äußerung behalten wird, ist nicht, dass sie ausgesprochen wird, sondern dass sie ein Ereignis ist, ein Diskursereignis, die Diskursinstanz, wie Benveniste sagt“"

99 Ebd. Die Welt ist jene der Leser. Und das Subjekt ist der Leser.

100 A. a. O.: „In der Interpretation wird, so sagen wir, die Lektüre wie ein Sprechen. Ich sage nicht, sie wird ein Sprechen. Denn die Lektüre kommt niemals einem Austausch von Worten, einem Dialog gleich“.

101 Ebenda..

102 Das erklärt er am Ende seines Aufsatz (vgl. Ricoeur, ebd., S. 104-107). Es sei angemerkt, dass Ricoeur Peirce durch die Einführung von Granger (Essai d'une philosophie du style) gelesen hat (vgl. Ricoeur, ebd., 
von Aristoteles entspricht nicht einem zweiten (d.h. einem reflexiven) auf einen vorangegangenen reagierenden Diskurs (,nicht dem, was man in einer zweiten Sprache in Hinblick auf eine erste Sprache macht") sondern einer Eigenschaft des Diskurses selbst, welche die Funktion einer Vermittlung zwischen den Zeichen und der Welt übernimmt (,das, was bereits die erste Sprache macht, indem sie durch Zeichen unsere Beziehung zu den Dingen vermittelt" $)^{103}$. Es ist hier nicht der rechte Moment, auf die Ähnlichkeit mit Peirce einzugehen ${ }^{104}$, wichtig ist vor allem zu verstehen, dass der Einwand Ricoeurs gegen Aristoteles' Interpretationsbegriff logisch ist: Aristoteles macht innerhalb der Diskursivität keinen Unterschied zwischen der ausgesprochenen Sprache und dem Text (,Sie setzt nämlich eine Theorie der sprachlichen Äußerung und nicht des Textes voraus"). Obwohl Ricoeur von Aristoteles übernimmt, dass ,die Interpretation eine Interpretation durch die Sprache ist, bevor sie Interpretation ist, die sich auf Sprache bezieht ${ }^{* 105}$, hält er an seinem Widerstand gegen das Zusammenfallen von Interpretation und Diskurs fest. Auch wenn also die Interpretation innerhalb des Diskurses geschieht, ist doch nicht jeder Diskurs eine Interpretation. Hierauf beharrt Ricoeur mit Nachdruck bis zu seinem Aufsatz über die unvereinbaren Dimensionen der Diskursivität (Rhetorik, Poetik, Hermeneutik - s. u. 4). Es ist auch erwähnenswert, dass in dem Unterschied zwischen seiner eigenen Auffassung der Interpretation und der von Aristoteles die Reflexivität des Diskurses eine große Rolle spielt: um einen zweiten Diskurs über einen ersten halten zu können, muss zunächst die reflexive Fähigkeit der Sprache anerkannt werden (s. u. 6).

\subsection{Der Text als Paradigma der Distanzierung}

Zunächst muss man betonen, dass für Ricoeur der Text viel mehr ist als ein besonderer Fall der zwischenmenschlichen Kommunikation: der Text ist das Paradigma der Distanzierung in der Kommunikation. Damit „enthüllt“ der Text „einen fundamentalen Charakter der Geschichtlichkeit der menschlichen Erfahrung, nämlich dass sie Kommunikation in der Distanz und durch die Distanz ist“"106. Der Text bezeugt im Zentrum der Geschichtlichkeit der menschlichen Erfahrung selbst die positive und produktive Funktion der Distanzierung (im Gegensatz zu Gadamer, der sie nur als Verfremdung sieht). Deswegen ist der Text eine Kategorie des Diskurses und nicht der Schriftlichkeit ${ }^{107}$, und das Hauptproblem der Hermeneutik (bzw. der Interpretation) stammt nicht aus der Schriftlichkeit selbst, sondern aus

S. 106).

103 Vgl. Ricoeur, ebd., S. 105.

104 Bei der analogischen Anwendung zwischen dem Begriff der Interpretation (der Zeichen) von Peirce und seinem Begriff der Texte ist Ricoeur sehr „,vorsichtig“ (vgl. Ricoeur, ebd., S. 106-7): „Die offene Reihe der Interpretanten, die zu der Beziehung des Zeichens zum Gegenstand hinzukommt, fördert eine Dreiecksbeziehung zwischen Gegenstand, Zeichen und Interpretanten zutage, die als Modell für ein anderes Dreieck dienen kann, das sich auf der Ebene des Textes konstituiert. Der Gegenstand ist der Text selbst, das Zeichen ist die von der strukturellen Analyse freigelegte Tiefensemantik, und die Reihe der Interpretanten ist die Kette der Interpretationen, die von der interpretierenden Gemeinschaft hervorgebracht werden und als Arbeit des Sinns an sich selbst in die Dynamik des Textes eingegliedert werden. In dieser Kette dienen die ersten Interpretanten als Tradition für die letzten, welche die eigentliche Interpretation sind“.

105 Ebenda, S. 106. Eigentlich ist „,die Interpretation im Aristotelischen Sinn nicht genau auf das Verständnis dieser dynamischen Beziehung zwischen mehreren Bedeutungsschichten des gleichen Textes vorbereitet" (ebd., S. 105).

106 Im Original: „La fonction herméneutique de la distanciation”, in Du texte à l'action, a. a. O., S. 114 : le texte „révèle un caractère fondamental de l'historicité même de l'expérience humaine, à savoir qu'elle est communication dans et par la distance“.

107 Darauf besteht Ricoeur, auch wenn er manchmal die beiden Wörter als Synonyme benutzt. Siehe z.B. ebd., S. 114: „on ne saurait (...) identifier purement et simplement texte et écriture“. Deshalb sagt er, dass die Hermeneutik die Kunst ist, in dem Werk (und auch im Text) den Diskurs auszumachen (,,'art de discerner le discours dans l'oeuvre“, ebd., S. 124). 
der Dialektik zwischen Sprachlichkeit und Schriftlichkeit (und ursprünglich aus der zwischen Ereignis und Bedeutung).

Vor diesem Hintergrund lässt sich auch die Aufgabe der Hermeneutik betrachten. Die Hermeneutik soll einerseits im Text selbst nach der inneren werkaufbauenden Dynamik suchen und anderseits nach dem Vermögen des Werks, sich außerhalb seiner selbst zu projizieren und somit eine durch den Text erschaffene Welt zu kreieren. Die Aufgabe für die Hermeneutik ist eine doppelte: (1) Sie muss einerseits sowohl der Gleichsetzung des Verstehens mit der Intropathie als auch der Reduzierung der Erklärung auf abstrakte Kombinationen widerstehen. Die Hermeneutik muss vielmehr in einer Dialektik zwischen dem Erklären und dem Verstehen zum „Sinn“ eines Werks führen. Die begründete Unterscheidung zwischen Sinn und Referenz ermöglicht es Ricoeur, sich Heideggers und Gadamers strenger und vollkommener Unterordnung der Epistemologie unter die Ontologie $\mathrm{zu}$ entziehen. Durch diese Definition von Hermeneutik wird ihre Beziehung zu den Geisteswissenschaften nachvollziehbar. (2) Vor dem Hintergrund der neuen hermeneutischen Ontologie Heideggers wird andererseits eindeutig die Beziehung der Sprache zur Welt unterstrichen, sogar für die poetische Sprache, für deren Besonderheit Ricoeur den Begriff der verdoppelten Referenz einführt.

\section{Die Ebenen des Sinnes}

Ricoeur betont bei einigen Gelegenheiten wie wichtig es sei, die verschiedenen Ebenen der Diskursivität auseinander zu halten. Die Ebene des Wortes ist für ihn keine echte Ebene der Sinnbildung. Während sie aber sowohl von der traditionellen aristotelischen Rhetorik als auch im französischen Strukturalismus nach Saussure als Bedeutungsatom betrachtet wird, beharrt Ricoeur darauf, dass die authentische Sinnbildung mit dem Satz beginnt. In der Behandlung dieses Themas wurde er von Benveniste (und seiner „Sprachwissenschaft des Ausdrucks“"), aber auch von G. Guillaume, Chomsky (die Performanz) und Jakobson beeinflusst ${ }^{108}$, von denen er lernte, das Sprechen als Handlung zu betrachten. Der Satz besteht aus einer prädikativen Funktion (oder Handlung), durch die einem Ding Eigenschaften oder Qualitäten zugesprochen werden (oder wodurch dieses Ding einer Kategorie zugeordnet) und einer Identifizierungsfunktion, durch die ein einzelnes Ding genannt wird ${ }^{109}$. Um die verschiedenen Handlungsaspekte des Satzes zu verstehen, greift Ricoeur auf Autoren wie Austin und Searle zurück (s. o.).

Der Satz stellt den Beginn der Ebene der Diskursivität im weiteren Sinne dar. Doch erklärt Ricoeur, dass etwas mit dem Sinn geschieht, wenn mehrere Sätze zusammengeschlossen werden. So ist der Diskurs im engeren Sinn in der Tat eine Sequenz von mehreren Sätzen, und zwar eine „Rede (..), also [ein] Sprechakt (..), deren Umfang demjenigen des Satzes entspricht oder darüber hinausgeht ${ }^{\star 110}$ - deswegen auch als trans-

108 Das unterstreicht er, für Benveniste und Jakobson, in „Eine intellektuelle Autobiographie” in: Vom Text zur Person, ebd., S. 32: „(...) die Tatsache, dass die erste Sinneinheit der lebendigen Sprache nicht das lexikalische Zeichen, sondern der Satz ist, den er Diskursinstanz nannte“. Zum Einfluss von Chomsky und Guillaume, siehe „Die Struktur, das Wort und das Ereignis“ in Hermeneutik und Strukturalismus, ebd., S. 113-116.

109 Siehe auch „Die Metapher und das Hauptproblem der Hermeneutik“, in Vom Text zur Person, ebd., S. 113: Ricoeur spricht von dem inneren Gegensatz der Proposition ,zwischen einem Pol der singulären Identifizierung (dieser Mann, dieser Tisch, Monsieur Dupont, Paris) einerseits und einem Pol der allgemeinen Prädikation (Menschheit als Klasse, Klarheit als Eigenschaft, Gleichheit als Relation, Laufen als Handlung)“. Diese Idee übernimmt er von P. Strawson, Individuals, London, Methuen, 1959. Dieses Buch ließ Ricoeur beim Verlag Seuil übersetzen (1973). Die grosse Bedeutung von Strawson wurde von Ricoeur in Das Selbst als ein Anderer unterstrichen (erste Abhandlung) und auch an anderer Stelle betont (siehe „Rhétorique, poétique, herméneutique“, in: Lectures 2, ebd., S. 481.

110 Vgl. Zeit und die historische Erzählung, ebd., incipit. Es muss bemerkt werden, dass Rainer Rochlitz 
phrastisch bezeichnet. Den Text als eine „semantische Einheit, die größer als der Satz ist“, betrachtet Ricoeur als eine bestimmte Sinnbildungsebene. Wie Ricoeur in seiner intellektuellen Autobiographie schreibt: „Mit dem Text erscheinen über den Satz hinausgehende Regeln der Komposition, die sich nicht auf die prädikative Operation beschränken, die nach E. Benveniste für die Diskursinstanz kennzeichnend ist: Die Fabelkomposition wird die bevorzugte Veranschaulichung dieser Kompositionsregeln sein"“111.

Kürzlich schrieb Ricoeur, dass es außer dem Text noch eine weitere Ebene gebe, und zwar die Kultur als Textzusammenhang: ,die erste Einheit der bedeutenden Sprache ist der Satz und keinesfalls das Wort [...] die Sätze sind kleine Diskurse, die längeren Diskursen, den Texten, entnommen sind (...) und die Texte gehören ihrerseits zu Kulturgebilden, innerhalb derer verschiedene Weltbilder zum Ausdruck kommen, und die sich übrigens innerhalb desselben Grundsystems phonologischer, lexikalischer oder syntaktischer Untergliederung so weit bekämpfen können, dass aus der sogenannten nationalen oder gemeinschaftlichen Kultur ein Netzwerk offen oder versteckt miteinander wettstreitender Weltbilder wird; es sei nur an den Okzident erinnert, an die Abfolge dessen, was zu seiner Bildung beigesteuert hat, an die griechischen, lateinischen und hebräischen Beiträge. Erinnern wir uns auch an die Perioden, in denen verschiedene Selbstverständnisse, in Mittelalter, Renaissance, Reformation, Aufklärung und Romantik miteinander rivalisierten“. ${ }^{112}$

\subsection{Bemerkung über die Metapher (als falsche Ebene der Sinnbildung)}

Es wurde schon gesagt, dass sich das wichtigste Phänomen der Mehrdeutigkeit nicht auf der Ebene des Symbols befindet, wie Ricoeur zuerst dachte, sondern auf der des Textes. Man muss diesen Aspekt etwas präzisieren, schließlich hat Ricoeur ein ganzes Buch über das Problem der Mehrdeutigkeit der Metapher geschrieben, welches bei ihm auf der Satzebene angesiedelt ist. So stellt sich dann die Frage, ob es sich bei dem Satz um eine echte Sinnebene handelt oder nicht. Diese Frage ist umso bedeutender als in Ricoeurs Theorie (s. o.) der Satz, im Gegensatz zum Wort, als authentischer Produktionsort des Sinns genannt wird. Eigentlich zeigt Die Lebendige Metapher, dass die Schwierigkeit, die Metapher auf der Wortebene zu erklären, (d.h. der Versuch der traditionellen Rhetorik, die Metapher als unrichtige Benennung zu bezeichnen) zu einer Aporie führt. Deshalb soll die Metapher auch auf der Satzebene verstanden werden, und zwar als unpassende Prädikation (,prédication impertinente" $)^{113}$. Trotz dieses Fortschritts lässt er sich nicht auf die hermeneutische Ebene (d. h. die diskursive Ebene, die für Gedichte, Erzählungen, usw. gilt) festlegen - denn eigentlich geht es für Ricoeur nur darum, die Wichtigkeit der Referenz (außerhalb (?) des

„discours“ als „Rede“ übersetzt. Meiner Meinung nach ist diese(r) )Übersetzung nicht richtig, wie erklärt wurde (s. o.), und man sollte die Übersetzung von Peter Welsen (,Diskurs“) benutzen.

Siehe „Eine intellektuelle Autobiographie”, in Vom Text zur Person, ebd. S. 42.

112 Vgl. „Un ,passage' : traduire l'intraduisible“, in Sur la traduction, Paris, Bayard, 2004, S. 55-6 : „,...) la première unité de langage signifiant est la phrase et non le mot (...) les phrases sont de petits discours prélevés sur de plus longs discours qui sont des textes (...) et les textes à leur tour font partie d'ensembles culturels à travers lesquels s'expriment des visions du monde différentes, qui d'ailleurs peuvent s'affronter à l'intérieur du même système élémentaire de découpage phonologique, lexical, syntaxique, au point de faire de ce qu'on appelle la culture nationale ou communautaire un réseau de visions du monde en compétition occulte ou ouverte ; pensons seulement à l'Occident et à ses apports successifs, grec, latin, hébraïque, et à ses périodes d'auto-compréhensions compétitives, au Moyen-Age, à la Renaissance et à la Réforme, aux Lumières et au Romantisme“. In Où vont les valeurs ? (J. Bindé (Hrsg.), Paris, Unesco-Albin Michel, 2004), spricht auch Ricoeur von einer ähnlichen asiatischen Übersetzungstradition des „radikal Anderen“, von einem vergleichbaren Ausmaß: die Übersetzung von indianischen buddhistischen Texten in China und danach in Japan und noch später in ganz Süd-Ost Asien.

113 Siehe Die Lebendige Metapher, ebd., Vorrede, S. 8. 
semantischen Sinns) zu bestätigen. Genauer gesagt, bleibt die Definition der Metapher als „unpassende Prädikation“ auf der Satzebene, d. h. auf der phrastischen stehen, ohne dass die transphrastische, diskursive Ebene erforscht wird. Ricoeur beginnt durch seine Untersuchung der Narrativität (Erzählung) diese diskursive Dimension methodisch zu studieren, wobei ihn die deutschen Theoretiker der so genannten Rezeptionsästhetik wie Jauss und andere Autoren, z.B. Weinrich, sehr beeinflussen ${ }^{114}$.

\section{Dimensionen der Diskursivität}

Im Allgemeinen lassen sich im Diskurs die Rhetorik, die Poetik und die Hermeneutik unterscheiden. Dabei ist es eminent wichtig, die Hermeneutik nur als eine von mehreren Dimensionen der Diskursivität zu betrachten. Dieser Punkt ist umso bedeutender, als Ricoeur sehr oft ausschließlich als Denker der Hermeneutik gesehen wird, obwohl er doch auch viel über die Poetik geschrieben hat ${ }^{115}$. Und wenn er auch nicht so viel über die Rhetorik geschrieben hat, kennt er doch die Werke zur Argumentation von Stephen Toulmin, Chaïm Perelman, K. O. Apel und Jürgen Habermas sehr gut ${ }^{116}$.

Die Rhetorik, sei sie auf das genus deliberativum, iudicale oder demonstrativum bezogen, entspricht einer auf Wahrscheinlichkeit bedachten Beweisführung, die auf eine Zuhörerschaft ausgerichtet ist. Die Poetik entspricht der Konfiguration, d.h. der Erzählungserfindung durch die kreative (produktive) Phantasie, die zur gleichen Zeit eine „Refiguration“ des menschlichen Praxisfeldes ist: Sie kann sogar zur politischen Utopie führen, doch erlaubt es ihre Vorstellungsnatur ihr nicht, zu begrifflicher Strenge zu gelangen. Die Hermeneutik entspricht der Interpretationstheorie von verschiedenen Textbedeutungen. Die Hermeneutik, sei sie nun religiös, philologisch oder rechtlich, stützt sich auf die kreative Phantasie, um die Textwelt mit der Realität zu verbinden und damit diese Realität wiederum darzustellen. Die Kreativität der Hermeneutik wird von der Ideologiekritik korrigiert. Jede dieser drei Disziplinen ist unvereinbar mit den anderen: es gibt einzig Treffpunkte der drei Sphären, jedoch keine diskursive Totalisierung ${ }^{117}$.

Dieselbe Idee des Diskursfeldes wurde danach weitergeführt und Ort der ,,praktischen Klugheit” genannt, dessen Wahrscheinlichkeits- Subsumptions- (oder Anwendungs-) und Erfindungseigenschaften dem kantischen Reflexionsurteil entsprechen ${ }^{118}$.

114 Vgl. Sur la traduction, a. a. O., S. 46-: „Wessen bedienen wir uns, wenn wir mit jemandem sprechen, das Wort an einen Anderen richten? Wir bedienen uns dreier Arten von Einheiten: der Wörter, d.h. der Zeichen, die in Wortverzeichnissen aufgeführt sind, der Sätze, für die es kein Verzeichnis gibt (niemand kann sagen wie viele Sätze im Französischen oder in jeder anderen Sprache ausgesprochen worden sind, und noch in Zukunft ausgesprochen werden) und schließlich der Texte, d.h. der Satzsequenzen (...) drei Typen von Einheiten: die eine wurde von Saussure aufgezeigt, die nächste von Benveniste und Jakobson und die dritte von Weinrich, Jauss und den Theoretikern der Textrezeption“. (,Avec quoi travaillons-nous quand nous parlons et adressons la parole à un autre ? Avec trois sortes d'unité : les mots, c'est-à-dire les signes qu'on trouve dans le lexique, les phrases, pour lesquelles il n'y a pas de lexique (nul ne peut dire combien de phrases ont été et seront dites en français ou en toute autre langue), et enfin les textes, c'est-à-dire des séquences de phrases (...) trois sortes d'unités, l'une pointée par Saussure, l'autre par Benveniste et Jakobson, la troisième par Harald Weinrich, Jauss et les théoriciens de la réception des textes“).

115 In Zeit und Erzählung (und Die lebendige Metapher) studiert Ricoeur das Phänomen der semantischen Innovation, die eine gute Definition der Poetik ist. Siehe auch „Une reprise de La Poétique d'Aristote“ (1992), in Lectures 2, Paris, Seuil, 1999.

116 S. Toulmin, The Use of Argument; C. Perelman, Traité de l'argumentation; Apel \& Habermas, Diskursethik. Siehe auch „Langage politique et rhétorique“ (1990), in Lectures 1, Paris, Seuil, 1991. Ricoeur kritisiert die Idee von Perelman, dass die Rhetorik ein Feld sei, das bis zur Philosophie reicht (L'Empire rhétorique, Paris, Vrin, 1997).

117 Siehe „Rhétorique, poétique, herméneutique”, in Lectures 2, a. a. O., S. 495 : „Argumenter, configurer, redécrire, telles sont les trois opérations majeures que leur visée totalisante respective rend exclusives l'une de l'autre, mais que la finitude de leur site originel condamne à la complémentaritéc.

118 Siehe Le Juste 1, Paris, Esprit, S. 25. 


\section{Die Rolle der Reflexivität und die analogische Einheit der Diskursivität}

Bei der Hermeneutik handelt es sich, wie schon gesagt wurde, um einen zweiten Diskurs, der über einen ersten spricht (quasi ein Kommentar des ersten ist). Die Möglichkeit dieser Übernahme ${ }^{119}$ liegt im tiefsten Innern der Diskursivität, bzw. in der Reflexivität begründet. Ricoeur versucht diese Eigenschaft der Sprache in der Übersetzung aufzuzeigen. In Sur la traduction "bemueht sich Ricoeur, zwei Arten der Übersetzung zu unterscheiden. Neben der ersten, der ,äußeren“ Übersetzung, die von einer Sprache in eine andere (z.B. FranzösischDeutsch) übertragen wird, gibt es noch eine ,innere“ Form - was die Methode betrifft, führt die ,äußere“ zur Entdeckung der zweiten. Diese „,innere“ Übersetzung besteht darin, innerhalb der gleichen Sprache „das Gleiche anders zu sagen“ (definieren, erklären, neu formulieren, usw.). Diese Paraphrasierung bestätigt Ricoeur als festes Sprachfaktum ${ }^{120}$, dessen Möglichkeitsbedingung in der reflexiven Fähigkeit der Sprache liegt, also in der Möglichkeit über Sprache zu sprechen und unsere Sprache als eine unter vielen zu betrachten ${ }^{121}$.

Die Reflexivität steht im Zusammenhang mit der Hermeneutik. In seinem Buch Über Freud versucht Ricoeur eine transzendentale Logik der Mehrdeutigkeit zu entwickeln ${ }^{122}$, in der die Reflexion durch die Vermittlung von Texten (und damals auch von Symbolen) geschehen soll, und die Mehrdeutigkeit der Texte (und der Symbole) die Reflexion zur Eindeutigkeit bringt. Die Mehrdeutigkeit zu reduzieren, läuft auf einen Konflikt der Interpretationen hinaus, die die reflektierende Urteilskraft stimulieren ${ }^{123}$ - wegen der Polysemie kann keine allgemeine Hermeneutik geschaffen werden. Deswegen kann man auch, kurz gesagt, die Hermeneutik als die reflexive Antwort auf die Mehrdeutigkeit, die das transphrastische Niveau des Diskurses in Form eines Textes ausbreitet, definieren.

Von einem logischen Standpunkt aus betrachtet, ist nicht die Verbindung der sprachlichen Reflexivität, einer reinen Eigenschaft des Symbolismus, mit der effektiven Reflexion des Menschen als Selbst (gebrochenes cogito) ${ }^{124}$ wichtig, sondern der Gedanke, dass der Diskurs

119 Vgl. „Was ist ein Text?”, in Vom Text zur Person, a. a. O., S. 108: „Das Sagen des Hermeneutikers ist ein erneutes Sagen, welches das Sagen des Textes reaktiviert".

120 Siehe Sur la traduction, ebd., S. 45: „Ich gehe von jenem gewichtigen Charakteristikum unseres Sprachgebrauches aus, dem Umstand nämlich, dass es immer möglich ist die gleiche Sache anders zu sagen“ (,Je partirai de ce fait massif caractéristique de l'usage de nos langues : il est toujours possible de dire la même chose autrement").

121 Vgl. Sur la traduction, a. a. O., S. 24-5 : “die Kennzeichen der intralinguistischen Übersetzung, das heisst [...] die Reflexionsfähigkeit der Sprache als immer bestehende Möglichkeit über Sprache zu reden, zu ihr auf Distanz zu gehen und damit unsere eigene Sprache wie eine beliebige Sprache unter anderen zu behandeln“ ([...] les traits de traduction intra-linguistiques, à savoir [...] la capacité réflexive du langage, cette possibilité toujours disponible de parler sur le langage, de le mettre à distance, et ainsi de traiter notre propre langage comme une langue parmi d'autres“). In einer ähnlicher Weise spricht Benveniste von einer metalinguistischen Fähigkeit der Sprache, uns über die Sprache zu erhöhen, uns von ihr zu abstrahieren, sie zu beobachten, und zur gleichen Zeit dieselbe Sprache für unsere Argumente und Bemerkungen zu benutzen („La forme et le sens dans le langage“, in Probleme der allgemeinen Sprachwisschenschaft, ebd.).

122 Siehe Die Interpretation, a. a. O., Problematik, III- die hermeneutische Methode und die reflexive Philosophie.

123 Ricoeur insistiert über die Reziprozität zwischen der Reflexion und der Mehrdeutigkeit - daher die transzendentale Dimension der Logik der Polysemie, die ,die verschiedenen konfliktuellen Interpretationen zusammen in der Reflexion verwurzeln“ (vgl. ebd., S. 66).

$124 \mathrm{Zu}$ diesem Thema der notwendigen Vermittlung des Cogito, die auch zentral in Das Selbst als ein Anderer ist, wird Ricoeur insbesondere von Gabriel Marcel und seiner Idee der „,konkreten“ Reflexion inspiriert. Über diese existentialistische Herkunft der Philosophie von Ricoeur, siehe „Réflexion primaire et réflexion seconde chez Gabriel Marcel“ (1984), in Lectures 2, ebd., S. 49 (und auch Gabriel Marcel et Karl Jaspers. Philosophie du mystère et philosophie du paradoxe, Paris, Seuil, 1948). Vgl. auch „Was ist ein Text?“, in: Vom Text zur Person, ebd., S. 99: „Diese Vollendung des Verständnisses des Textes in einem Selbstverständnis kennzeichnet die Art der Reflexionsphilosophie, die ich bei verschiedenen Gelegenheiten „konkrete Reflexion“ genannt habe. Hermeneutik und Reflexionsphilosophie sind hier korrelativ und 
keine definitive Einheit haben kann. Seine Einheit kann nur durch die Reflexivität analogisch betrachtet werden. Wenn also keine der Diskursdimensionen (Rhetorik, Poetik, Hermeneutik) die Diskursivität selbst definieren kann, so spielt aber die Reflexivität die Rolle einer relativen, $d . h$. analogischen Vereinigung dieser Diskursivität. Nur sie ermöglicht es, den Diskurs von verschiedenen Standpunkten aus zu beobachten bzw. ihn als jeweils poetisch, argumentativ, hermeneutisch zu betrachten. Doch sie selbst ist leer und muss sich stets erst noch vermittels dieser drei Dimensionen die Diskursivität konstituieren. Daher stammen auch die Plastizität des Diskurses und die Unmöglichkeit einer endgültigen reflexiven Zusammenfassung. Weil der Diskurs nie genau mit sich selbst zusammenfallen kann (wie es bei dem Begriff in der Logik Hegels der Fall ist - beim Absoluten als das sich denkende diskursive Subjekt), bewegt und verändert er sich immer wieder, um sich selbst stets neu auszusagen $^{125}$.

\section{Schluss}

Die(se) hier dargelegte logische Untersuchung des Diskursbegriffes bei Ricoeur könnte in zwei verschiedenen Richtungen weiter geführt werden. Zunächst könnte man erklären, wie auf dem Diskurs eine vollständige Philosophie der Handlung aufbaut, und zwar eine Theorie der symbolisch vermittelten Handlung (nach C. Geertz), die das sinnhaft(e) orientierte Verhalten (nach Weber) als einen Text betrachtet ${ }^{126}$. Die Logik der Praxis kann als Anwendung der diskursiven Logik auf die Praxis studiert werden. Unter diesem Blickwinkel lassen sich auch viele Bemerkungen Ricoeurs über die Rechtsphilosophie verstehen ${ }^{127}$, u.a. die Bedeutung, die er der Untersuchung der dialektischen Beziehung von Interpretation und Argumentation im juristischen Diskurs beimisst ${ }^{128}$.

reziprok. Einerseits durchläuft das Selbstverständnis den Umweg über das Verständnis der Zeichen der Kultur, in denen sich das Selbst kundtut und bildet, andererseits ist das Verständnis des Textes kein Selbstzweck, es vermittelt seine Beziehung zu einem Subjekt, das nicht im Kurzschluss einer unmittelbaren Reflexion den Sinn seines eigenen Lebens findet“. Zum Thema der Reflexion wurde Ricoeur auch von dem „fichtischen“ Philosophen Jean Nabert sehr beeinflusst: siehe „De l'interprétation“, in Du texte à l'action, a. a. O., S. 29; und auch Das Selbst als ein Anderer, ebd., Personenregister.

125 Ich würde das Schlusswort des „Avertissement“ von Réflexion faite auf folgende Art und Weise verstehen: „,der Ausdruck ,nach reiflicher Überlegung' darf nicht mit der Sentenz, alles in allem betrachtet' verwechselt werden. Die Überlegung schliesst, selbst in ihrer intensivierten Form, niemals etwas ab, sie zieht keine Bilanz" (l'expression "réflexion faite" (...) ne doit pas être confondue avec la sentence "tout compte fait". La réflexion, même redoublée, ne se referme pas sur un bilan"). Vgl., ebd., S. 8.

$126 \mathrm{Zu}$ diesem Thema, siehe das Buch Du texte à l'action, a. a. O., II: „De l'herméneutique des textes à l'herméneutique de l'action“, S. 153-310; und besonders den Artikel: „Le modèle du texte : l'action sensée considérée comme un texte", ebd., S. 205-236.

127 Vgl. Lectures 1, a. a. O.; Le juste 1 und Le juste 2 (ebenda); und Wege der Anerkennung (übers. aus dem französischen von Ulrike Bokelmann und Barbara Heber-Schärer), Suhrkamp Verlag, Frankfurt/Main, 2006.

128 Siehe „Interprétation et/ou argumentation“, in Le Juste 1, a. a. O., S. 163-184. Einerseits wird gegen Dworkin auf die Argumentation, und andererseits gegen Habermas auf die Interpretation mit Nachdruck bestanden. 